\title{
Oxidative Ring-Opening of Benzothiazole Derivatives
}

\author{
Ryan E. Moreira, Geoffrey S. Sinclair, Derek J. Schipper \\ Department of Chemistry, University of Waterloo, Waterloo, Ontario N2L 3G1, Canada
}

\section{General Methods:}

Solvents were either reagent grade and/or HPLC grade. Chemical reagents were purchased from Sigma-Aldrich. Reactions were monitored using commercial thin-layer chromatography (TLC) plates. Developed TLC plates were examined under a UV lamp (254 nm). Flash chromatography was performed using 230-400 mesh silica gel. ${ }^{1} \mathrm{H}-\mathrm{NMR}$ spectra were recorded on a Brüker AVANCE300 $(300 \mathrm{MHz}) \delta$ or Brüker AC300 $(300 \mathrm{MHz}) \delta \mathrm{NMR}$ spectrometers. ${ }^{13} \mathrm{C}-\mathrm{NMR}$ spectra were broad band decoupled and recorded on a Brüker AVANCE300 $(75.5 \mathrm{MHz}) \delta$ or Brüker AC300 $(75.5 \mathrm{MHz}) \delta$ NMR spectrometers using the carbon signal of the deuterated solvent as the internal standard. The following abbreviations are used for NMR peak multiplicities: s, singlet; d, doublet; t, triplet; q, quartet; dd, doublet of doublets; dt, doublet of triplets; m, multiplet; br, broad. Chemical shifts are reported in parts per million (ppm) relative to either TMS $(\delta 0.0)$ or chloroform $(\delta 7.25)$ for ${ }^{1} \mathrm{H}-\mathrm{NMR}$, and chloroform $(\delta 77.0)$ for ${ }^{13} \mathrm{CNMR}$. High resolution mass spectra (HRMS) were obtained via electrospray ionization (ESI) or direct analysis in real time (DART) which were measured on a Thermo Scientific Q ExactiveTM Plus Hybrid Quadrupole-OrbitrapTM at the University of Waterloo Mass Spectrometry Facility. X-ray crystal structure was determined by Dr. Jalil Assoud and figures of Xray crystal structures were generated using Mercury.

\section{Contents}

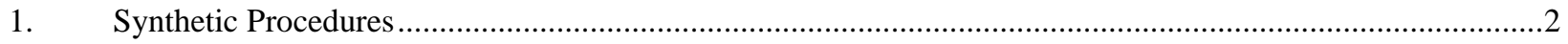

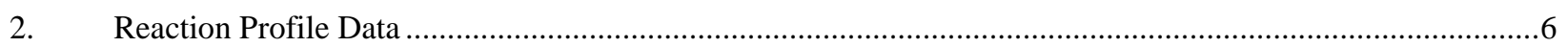

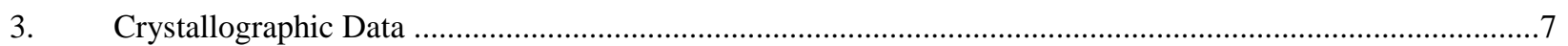

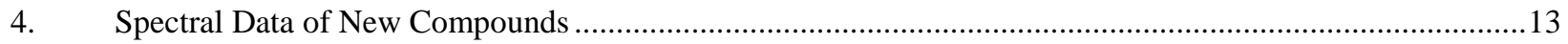




\section{Synthetic Procedures}

\section{GENERAL PROCEDURE A}

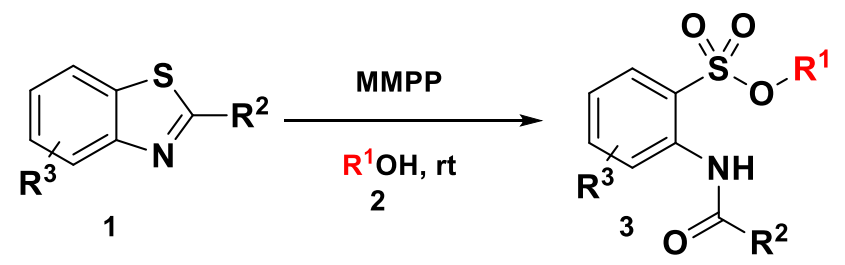

A $0.2 \mathrm{M}$ solution of benzothiazole $1(1.00 \mathrm{mmol}, 1.0$ equiv) in alcohol $2(5 \mathrm{~mL})$ was stirred vigorously, at which point MMPP (1.00 g, $1.60 \mathrm{mmol}, 1.6$ equiv) was added. The reaction mixture was stirred at room temperature for 4 hours. The reaction mixture was stirred at room temperature for 4 hours. After the reaction time, $\mathrm{H}_{2} \mathrm{O}(10 \mathrm{~mL})$ was added to the reaction vessel and the solution was extracted with $3 \times \mathrm{CH}_{2} \mathrm{Cl}_{2}(10 \mathrm{~mL})$. The organic layers were collected, dried over $\mathrm{MgSO}_{4}$, filtered and concentrated to yield the crude product. The product was purified by silica gel column chromatography (1:4 EtOAc/hexanes $\rightarrow 2: 3$ EtOAc/hexanes) to yield purified product 3 .

methyl 2-formamidobenzenesulfonate (3a)

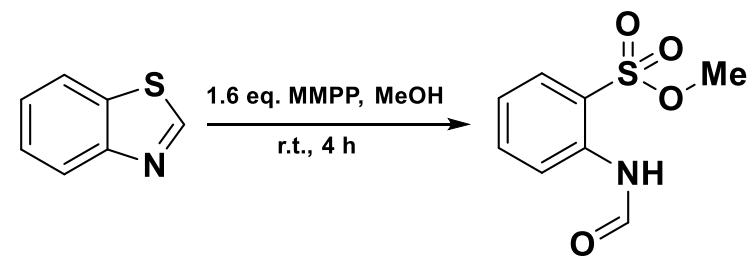

This compound was synthesized following the General Procedure A with benzothiazole 1a (135 mg) and MeOH (2a). This yielded 3a as a light yellow, waxy solid $(97 \mathrm{mg}, 0.45 \mathrm{mmol}, 45 \%$ yield $) . \mathrm{Rf}=0.4\left(2: 3 \mathrm{EtOAc/hexanes).}{ }^{1} \mathrm{H}\right.$ NMR $\left(\mathrm{CDCl}_{3}, 300 \mathrm{MHz}\right.$ ) (Rotamer ratio: 2.2:1, Asterisk denotes minor peak) $\delta 8.97$ (br. s, $\left.1 \mathrm{H}\right) * 8.53$ (br. s, $\left.1 \mathrm{H}\right) 8.59$ $(\mathrm{d}, 1 \mathrm{H}, J=8.58 \mathrm{~Hz}) 8.50(\mathrm{~s}, 1 \mathrm{H}) 7.93(\mathrm{~d}, 1 \mathrm{H}, J=7.99 \mathrm{~Hz}) 7.67(\mathrm{t}, 1 \mathrm{H}, J=7.81 \mathrm{~Hz}) 7.28(\mathrm{t}, 1 \mathrm{H}, J=7.80 \mathrm{~Hz}) 3.76(\mathrm{~s}$, $3 \mathrm{H}) .{ }^{13} \mathrm{C}$ NMR $\left(\mathrm{CDCl}_{3}, 100 \mathrm{MHz}\right) \delta 161.6,138.2,137.7,132.3,126.3,125.5,124.2,59.1$. HRMS calculated for $\mathrm{C}_{8} \mathrm{H}_{10} \mathrm{NO}_{4} \mathrm{~S}(\mathrm{M}+\mathrm{H})$ 216.0325; Found 216.0326.

ethyl 2-formamidobenzenesulfonate (3b)

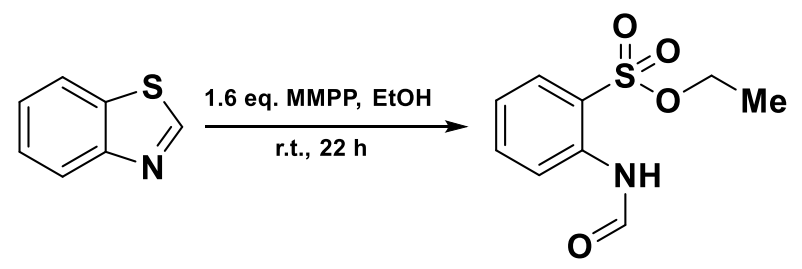

This compound was synthesized following the General Procedure A with benzothiazole 1a (135 mg), EtOH (2b) and a reaction time of $22 \mathrm{~h}$. This yielded $\mathbf{3 b}$ as a dull yellow solid $(105 \mathrm{mg}, 0.46 \mathrm{mmol}, \mathbf{4 6} \%$ yield $)$. $\mathrm{Rf}=0.28(1: 4$ EtOAc/hexanes). ${ }^{1} \mathrm{H}$ NMR $\left(\mathrm{CDCl}_{3}, 300 \mathrm{MHz}\right.$ ) (Rotamer ratio: 2.1:1, Asterisk denotes minor peak) $\delta 8.97$ (br. s, $\left.1 \mathrm{H}\right)$ *8.80 (br. s, $1 \mathrm{H}) 8.57(\mathrm{~d}, 1 \mathrm{H}, J=8.67 \mathrm{~Hz}) 8.48(\mathrm{~s}, 1 \mathrm{H}) 7.93(\mathrm{~d}, 1 \mathrm{H}, J=7.93 \mathrm{~Hz}) 7.66(\mathrm{t}, 1 \mathrm{H}, J=7.92) 7.29(\mathrm{t}, 1 \mathrm{H}, J$ $=7.84 \mathrm{~Hz}) 4.11(\mathrm{q}, 2 \mathrm{H}, J=6.89 \mathrm{~Hz}) 1.31(\mathrm{t}, 3 \mathrm{H}, J=7.02) .{ }^{13} \mathrm{C} \mathrm{NMR}\left(\mathrm{CDCl}_{3}, 100 \mathrm{MHz}\right) \delta 159.3,135.8,135.5,130.1$, 124.3, 123.4(4), 123.4(2), 68.1, 14.7. HRMS calculated for $\mathrm{C}_{9} \mathrm{H}_{12} \mathrm{NO}_{4} \mathrm{~S}(\mathrm{M}+\mathrm{H}) 230.0482$; Found 230.0482. 


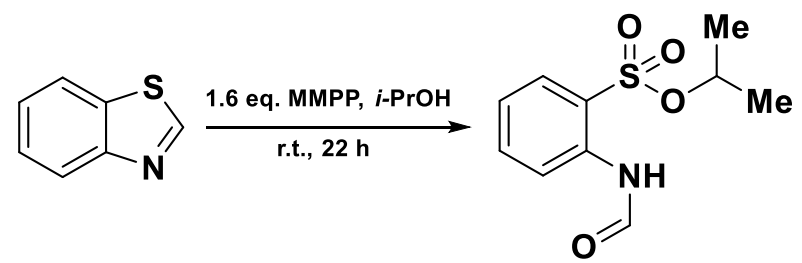

This compound was synthesized following the General Procedure A with benzothiazole 1a (135 mg), $i$-PrOH (2c) and a reaction time of $22 \mathrm{~h}$. This yielded $\mathbf{3 c}$ as a white solid $(57 \mathrm{mg}, 0.24 \mathrm{mmol}, \mathbf{2 5 \%}$ yield). $\mathrm{Rf}=0.38$ (2:3 EtOAc/hexanes). ${ }^{1} \mathrm{H} \mathrm{NMR}\left(\mathrm{CDCl}_{3}, 300 \mathrm{MHz}\right)$ (Rotamer ratio: 3.1:1, Asterisk denotes minor peak) $\delta 8.95$ (br. s, $\left.1 \mathrm{H}\right)$ *8.77 (br. s, 1H) $8.55(\mathrm{~d}, 1 \mathrm{H}, J=9.00 \mathrm{~Hz}) 7.94(\mathrm{~d}, 1 \mathrm{H}, J=7.50 \mathrm{~Hz}) 7.65(\mathrm{t}, 1 \mathrm{H}, J=7.81 \mathrm{~Hz}) 7.28(\mathrm{~m}, 1 \mathrm{H}) 4.70$ (sept., $1 \mathrm{H}, J=6.38 \mathrm{~Hz}) 1.26(\mathrm{~d}, 6 \mathrm{H}, J=6.12 \mathrm{~Hz}) .{ }^{13} \mathrm{C} \mathrm{NMR}\left(\mathrm{CDCl}_{3}, 100 \mathrm{MHz}\right) \delta 159.2,135.6,135.3,129.8,124.8,124.4$, 123.5, 79.0, 22.8. HRMS calculated for $\mathrm{C}_{10} \mathrm{H}_{13} \mathrm{NO}_{4} \mathrm{~S}(\mathrm{M}+\mathrm{H})$ 244.0638; Found 244.0638.

butyl 2-formamidobenzenesulfonate (3d)

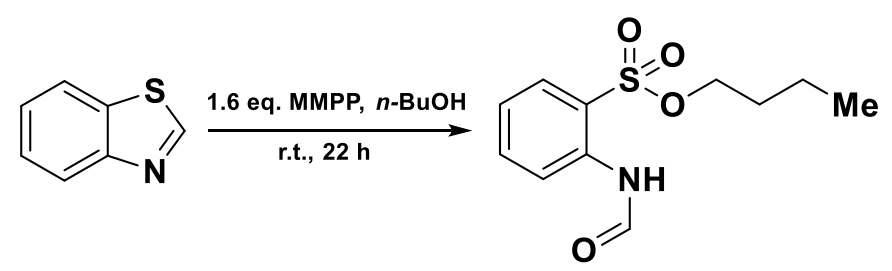

This compound was synthesized following the General Procedure A with benzothiazole 1a (135 mg), $n$-BuOH (2d) and a reaction time of $22 \mathrm{~h}$. The butanol was removed in-vacuo using a toluene:butanol (1:1) azeotrope. This yielded 3d as a bright yellow solid (77 mg, $0.30 \mathrm{mmol}, \mathbf{3 0 \%}$ yield $) . \mathrm{Rf}=0.30(1: 5 \mathrm{EtOAc} /$ hexanes $) .{ }^{1} \mathrm{H}$ NMR $\left(\mathrm{CDCl}_{3}, 300\right.$ MHz) (Rotamer ratio: 2.0:1, Asterisk denotes minor peak) $\delta 9.00$ (br. s, $1 \mathrm{H}) * 8.79$ (br. s, $1 \mathrm{H}) 8.57$ (d, $1 \mathrm{H}, J=8.69$ $H z) 8.50(\mathrm{~s}, 1 \mathrm{H}) 7.92(\mathrm{~d}, 1 \mathrm{H}, J=7.72 \mathrm{~Hz}) 7.66(\mathrm{t}, 1 \mathrm{H}, J=7.93 \mathrm{~Hz}) 7.28(\mathrm{t}, 1 \mathrm{H}, J=7.72 \mathrm{~Hz}) * 4.10(\mathrm{~m}, 2 \mathrm{H}) 4.03(\mathrm{t}$, $2 \mathrm{H}, J=6.74 \mathrm{~Hz}$ ) 1.61 (quint, $2 \mathrm{H}, J=6.74 \mathrm{~Hz}$ ) 1.31 (sext, $2 \mathrm{H}, J=7.38 \mathrm{~Hz}$ ) $0.84\left(\mathrm{t}, 3 \mathrm{H}, J=7.34 \mathrm{~Hz}\right.$ ). ${ }^{13} \mathrm{C}$ NMR $\left(\mathrm{CDCl}_{3}, 100 \mathrm{MHz}\right) \delta 159.2,135.9,135.5,130.2,124.4,123.4,123.3,71.7,30.8,18.7,13.5$. HRMS calculated for $\mathrm{C}_{11} \mathrm{H}_{16} \mathrm{NO}_{4} \mathrm{~S}(\mathrm{M}+\mathrm{H})$ 258.0795; Found 258.0796.

\section{2,2,2-trifluoroethyl 2-formamidobenzenesulfonate (3e)}<smiles>O=CNc1ccc(O)cc1S(=O)(=O)OCC(F)(F)F</smiles>

This compound was synthesized following the General Procedure A with benzothiazole 1a (135 mg), 2,2,2trifluoroethanol (2e) and a reaction time of $22 \mathrm{~h}$. This yielded 3e as a light yellow solid (29 $\mathrm{mg}, 0.11 \mathrm{mmol}, \mathbf{1 1 \%}$ yield). $\mathrm{Rf}=0.38(2: 3 \mathrm{EtOAc} /$ hexanes $) .{ }^{1} \mathrm{H} \mathrm{NMR}\left(\mathrm{CDCl}_{3}, 300 \mathrm{MHz}\right)$ (Rotamer ratio: 5.4:1, Asterisk denotes minor peak) $\delta 8.82$ (br. s, $1 \mathrm{H}) * 8.76($ br. s, $1 \mathrm{H}) 8.62(\mathrm{~d}, 1 \mathrm{H}, J=8.36 \mathrm{~Hz}) 8.51(\mathrm{~s}, 1 \mathrm{H}) * 8.037(\mathrm{~m}, 1 \mathrm{H}) 7.93(\mathrm{~d}, 1 \mathrm{H}, J=8.07$ $\mathrm{Hz}) 7.72(\mathrm{t}, 1 \mathrm{H}, J=7.82 \mathrm{~Hz}) * 7.44(\mathrm{~m}, 1 \mathrm{H}) 7.31(\mathrm{t}, 1 \mathrm{H}, J=7.83 \mathrm{~Hz}) * 4.43(\mathrm{~m}, 2 \mathrm{H}) 4.36(\mathrm{q}, 2 \mathrm{H}, J=8.39 \mathrm{~Hz}) .{ }^{13} \mathrm{C}$ NMR $\left(\mathrm{CDCl}_{3}, 100 \mathrm{MHz}\right) \delta 158.9,136.4,136.1,130.0,125.7,124.4,123.6,122.6,121.7,120.4,118.2,65.4,65.1$, 64.8, 64.5. ${ }^{19} \mathrm{~F}$ NMR $\left(\mathrm{CDCl}_{3}, 280 \mathrm{MHz}\right) \delta$-73.8. HRMS calculated for $\mathrm{C}_{9} \mathrm{H}_{9} \mathrm{NO}_{4} \mathrm{SF}_{3}(\mathrm{M}+\mathrm{H})$ 284.0199; Found 284.0198 . 
ethyl 2-acetamidobenzenesulfonate (3h)<smiles>CCOS(=O)(=O)c1ccccc1NC(C)=O</smiles>

This compound was synthesized following the General Procedure A with 2-methylbenzothiazole $1 \mathbf{b}$ (149 mg), ethanol (2b) and a reaction time of $22 \mathrm{~h}$. This yielded $\mathbf{3 h}$ as a pale yellow solid $(43 \mathrm{mg}, 0.18 \mathrm{mmol}, \mathbf{1 8 \%}$ yield). $\mathrm{Rf}=0.24$ (1:4 EtOAc/hexanes). ${ }^{1} \mathrm{H}$ NMR $\left(\mathrm{CDCl}_{3}, 300 \mathrm{MHz}\right) \delta 8.96$ (br. s, $\left.1 \mathrm{H}\right) 8.46(\mathrm{~d}, 1 \mathrm{H}, J=8.38 \mathrm{~Hz}) 7.88$ (dd, $1 \mathrm{H}, J=7.98$, $1.45 \mathrm{~Hz}) 7.65-7.59(\mathrm{~m}, 1 \mathrm{H}) 7.21(\mathrm{t}, 1 \mathrm{H}, J=7.68 \mathrm{~Hz}) 4.08(\mathrm{q}, 2 \mathrm{H}, J=7.11 \mathrm{~Hz}) 2.22(\mathrm{~s}, 3 \mathrm{H}) 1.27(\mathrm{t}, 3 \mathrm{H}, J=7.12 \mathrm{~Hz})$. ${ }^{13} \mathrm{C} \mathrm{NMR}\left(\mathrm{CDCl}_{3}, 100 \mathrm{MHz}\right) \delta 168.8,137.0,135.3,129.9,123.7,123.4,123.2,68.0,25.2,14.8$. HRMS calculated for $\mathrm{C}_{10} \mathrm{H}_{14} \mathrm{NO}_{4} \mathrm{~S}(\mathrm{M}+\mathrm{H})$ 244.0644; Found 244.0639.

ethyl 2-ureidobenzenesulfonate (3i)<smiles>CCOS(=O)(=O)c1ccccc1NC(N)=O</smiles>

This compound was synthesized following the General Procedure A with 2-aminobenzothiazole 1c (149 mg), ethanol (2b), MMPP (3.2 equiv), and a reaction time of $22 \mathrm{~h}$. This yielded $3 \mathbf{i}$ as an orange solid (40mg, $0.16 \mathrm{mmol}, \mathbf{1 6 \%}$ yield). $\mathrm{Rf}=0.25(1: 1 \mathrm{EtOAc} /$ hexanes $) .{ }^{1} \mathrm{H} \mathrm{NMR}\left(\mathrm{CDCl}_{3}, 300 \mathrm{MHz}\right) \delta 7.90(\mathrm{~d}, 1 \mathrm{H}, J=7.1 \mathrm{~Hz}) 7.70(\mathrm{~d}, 1 \mathrm{H}, J=6.8$ $\mathrm{Hz}) 7.59-7.55(\mathrm{~m}, 2 \mathrm{H}) 5.98(\mathrm{br}, 3 \mathrm{H}), 4.38(\mathrm{q}, 2 \mathrm{H}, J=7.2 \mathrm{~Hz}) 1.37(\mathrm{t}, 3 \mathrm{H}, J=7.1 \mathrm{~Hz}) .{ }^{13} \mathrm{C} \mathrm{NMR}\left(\mathrm{CDCl}_{3}, 100 \mathrm{MHz}\right) \delta$ $171.9,168.2,133.3,132.0,130.9,130.3,129.9,128.8,62.0,13.9$. HRMS calculated for $\mathrm{C}_{9} \mathrm{H}_{13} \mathrm{~N}_{2} \mathrm{O}_{4} \mathrm{~S}(\mathrm{M}+\mathrm{H})$ 245.05905; Found 245.05909.

ethyl 4-bromo-2-formamidobenzenesulfonate (3j)<smiles>CCOS(=O)(=O)c1ccc(Br)cc1NC=O</smiles>

This compound was synthesized following the General Procedure A with 5-bromobenzothiazole 1d (214 mg), ethanol (2b) and a reaction time of $22 \mathrm{~h}$. This yielded $\mathbf{3 j}$ as a pale yellow solid $(100 \mathrm{mg}, 0.33 \mathrm{mmol}, \mathbf{3 3 \%}$ yield $)$. Rf $=0.25$ (1:4 EtOAc/hexanes). ${ }^{1} \mathrm{H}$ NMR $\left(\mathrm{CDCl}_{3}, 300 \mathrm{MHz}\right) \delta 9.01$ (br. s, $\left.1 \mathrm{H}\right) 8.82(\mathrm{~s}, 1 \mathrm{H}) 8.45(\mathrm{~s}, 1 \mathrm{H}) 7.75(\mathrm{~d}, 1 \mathrm{H}, J=8.44$ $\mathrm{Hz}) 7.40(\mathrm{~d}, 1 \mathrm{H}, J=8.44 \mathrm{~Hz}) 4.11(\mathrm{q}, 2 \mathrm{H}, J=7.02 \mathrm{~Hz}) 1.30(\mathrm{t}, 3 \mathrm{H}, J=7.06 \mathrm{~Hz}) .{ }^{13} \mathrm{C} \mathrm{NMR}\left(\mathrm{CDCl}_{3}, 100 \mathrm{MHz}\right) \delta$ 159.2, 136.7, 131.1, 130.5, 127.5, 126.0, 122.0, 68.4, 14.8. HRMS calculated for $\mathrm{C}_{9} \mathrm{H}_{10} \mathrm{BrNO}_{4} \mathrm{~S}(\mathrm{M}+\mathrm{H}) 307.9587$; Found 307.9586. 
<smiles>CCOS(=O)(=O)c1ccc([N+](=O)[O-])cc1NC=O</smiles>

This compound was synthesized following the General Procedure A with 5-nitrobenzothiazole 1e (180 mg), ethanol (2b) and a reaction time of $22 \mathrm{~h}$. This yielded 3k as a bright yellow solid $(52 \mathrm{mg}, 0.19 \mathrm{mmol}, \mathbf{1 9 \%}$ yield $)$. Rf $=0.42$ (2:3 EtOAc/hexanes). ${ }^{1} \mathrm{H}$ NMR $\left(\mathrm{CDCl}_{3}, 300 \mathrm{MHz}\right) \delta 9.31$ (br. s, $\left.1 \mathrm{H}\right) 8.91-8.89(\mathrm{~m}, 1 \mathrm{H}) 8.80(\mathrm{~d}, 1 \mathrm{H}, J=2.62 \mathrm{~Hz})$ 8.60 (br. s, $1 \mathrm{H}) 8.49(\mathrm{dd}, 1 \mathrm{H}, J=9.18,2.61 \mathrm{~Hz}) 4.25(\mathrm{q}, 2 \mathrm{H}, J=7.11 \mathrm{~Hz}) 1.37(\mathrm{t}, 3 \mathrm{H}, J=7.10 \mathrm{~Hz}){ }^{13} \mathrm{C} \mathrm{NMR}\left(\mathrm{CDCl}_{3}\right.$, $100 \mathrm{MHz}) \delta 159.1,142.6,140.8,130.1,125.7,123.7,123.0,69.1,14.7$. HRMS calculated for $\mathrm{C}_{9} \mathrm{H}_{11} \mathrm{~N}_{2} \mathrm{O}_{6} \mathrm{~S}(\mathrm{M}+\mathrm{H})$ 275.0338; Found 275.0333.

ethyl 2-formamido-5-nitrobenzenesulfonate (3l)<smiles>CCOS(=O)(=O)c1cc([N+](=O)[O-])ccc1NC=O</smiles>

This compound was synthesized following the General Procedure A with 6-nitrobenzothiazole 1f (180 mg), ethanol (2b) and a reaction time of $22 \mathrm{~h}$. This yielded $\mathbf{3 l}$ as a bright yellow solid $(57 \mathrm{mg}, 0.21 \mathrm{mmol}, \mathbf{2 1 \%}$ yield $)$. $\mathrm{Rf}=0.42$ (2:3 EtOAc/hexanes). ${ }^{1} \mathrm{H}$ NMR $\left(\mathrm{CDCl}_{3}, 300 \mathrm{MHz}\right) \delta 9.32$ (br. s, $\left.1 \mathrm{H}\right) 8.90(\mathrm{~d}, 1 \mathrm{H}, J=8.04) 8.80(\mathrm{~d}, 1 \mathrm{H}, J=2.05 \mathrm{~Hz}$ ) 8.59 (br. s, $1 \mathrm{H}) 8.49$ (dd, $1 \mathrm{H}, J=9.18,2.55 \mathrm{~Hz}) 4.24$ (q, $2 \mathrm{H}, J=7.05 \mathrm{~Hz}) 1.37$ (t, $3 \mathrm{H}, J=7.08 \mathrm{~Hz}) .{ }^{13} \mathrm{C} \mathrm{NMR}\left(\mathrm{CDCl}_{3}\right.$, $100 \mathrm{MHz}) \delta 159.1,142.6,140.8,130.1,125.7,123.7,123.0,69.2,14.7$. HRMS calculated for $\mathrm{C}_{9} \mathrm{H}_{11} \mathrm{~N}_{2} \mathrm{O}_{6} \mathrm{~S}(\mathrm{M}+\mathrm{H})$ 275.03323; Found 275.03350.

isopropyl 2-acetamidobenzenesulfonate (3n)<smiles>CC(=O)Nc1ccccc1S(=O)(=O)OC(C)C</smiles>

This compound was synthesized following the General Procedure A with 2-methylbenzothiazole $\mathbf{1 b}$ (149 mg), isopropanol (2c) and a reaction time of $22 \mathrm{~h}$. This yielded $\mathbf{3 n}$ as a dull yellow solid (17 $\mathrm{mg}, 0.07 \mathrm{mmol}, \mathbf{7 \%}$ yield). Rf $=0.17\left(1: 4 \mathrm{EtOAc} /\right.$ hexanes). ${ }^{1} \mathrm{H} \mathrm{NMR}\left(\mathrm{CDCl}_{3}, 300 \mathrm{MHz}\right)$ (Rotamer ratio: 7.8:1, Asterisk denotes minor peak) 8.92 (br. s, $1 \mathrm{H}) 8.43(\mathrm{~d}, 1 \mathrm{H}, J=8.44 \mathrm{~Hz}) * 8.01(\mathrm{~d}, 1 \mathrm{H}, J=7.94 \mathrm{~Hz}) 7.90(\mathrm{~d}, 1 \mathrm{H}, J=8.04 \mathrm{~Hz}) * 7.75(\mathrm{~m}, 1 \mathrm{H}) 7.62(\mathrm{t}, 1 \mathrm{H}$, $J=7.74 \mathrm{~Hz}) * 7.42(\mathrm{~m}, 1 \mathrm{H}) 7.22(\mathrm{t}, 1 \mathrm{H}, J=7.65 \mathrm{~Hz}) 4.66(\mathrm{sept}, 1 \mathrm{H}, J=6.28 \mathrm{~Hz}) 2.23(\mathrm{~s}, 3 \mathrm{H}) 1.24(\mathrm{~d}, 6 \mathrm{H}, J=6.23$ $\mathrm{Hz}) .{ }^{13} \mathrm{C} \mathrm{NMR}\left(\mathrm{CDCl}_{3}, 100 \mathrm{MHz}\right) 168.8,136.8,135.2,129.7,124.7,123.8,123.3,78.7,25.2,22.8$. HRMS calculated for $\mathrm{C}_{11} \mathrm{H}_{16} \mathrm{NO}_{4} \mathrm{~S}(\mathrm{M}+\mathrm{H})$ 258.0795; Found 258.0795. 
isopropyl 4-bromo-2-formamidobenzenesulfonate (3o)<smiles>CC(C)OS(=O)(=O)c1ccc(Br)cc1NC=O</smiles>

Chemical Formula: $\mathrm{C}_{10} \mathrm{H}_{12} \mathrm{BrNO}_{4} \mathrm{~S}$

This compound was synthesized following the General Procedure A with 5-bromobenzothiazole 1d (214 mg), ethanol (2c) and a reaction time of $22 \mathrm{~h}$. This yielded $\mathbf{3 o}$ as a dull yellow solid $(87 \mathrm{mg}, 0.19 \mathrm{mmol}, \mathbf{1 9 \%}$ yield $)$. $\mathrm{Rf}=0.38(1: 4$ EtOAc/hexanes). ${ }^{1} \mathrm{H} \mathrm{NMR}\left(\mathrm{CDCl}_{3}, 300 \mathrm{MHz}\right.$ ) (Rotamer ratio: 8.5:1, Asterisk denotes minor peak) $\delta 8.97$ (br. s, $\left.1 \mathrm{H}\right)$ $8.81(\mathrm{~s}, 1 \mathrm{H}) * 8.79$ (br. s, $1 \mathrm{H}) 8.49(\mathrm{~s}, 1 \mathrm{H}) 7.77(\mathrm{~d}, 1 \mathrm{H}, J=8.43 \mathrm{~Hz}) * 7.57(\mathrm{~m}, 1 \mathrm{H}) 7.41(\mathrm{~d}, 1 \mathrm{H}, J=8.26 \mathrm{~Hz}) * 4.82$ (m, 1H) 4.71 (sept., $1 \mathrm{H}, J=5.4 \mathrm{~Hz}) 1.27(\mathrm{~d}, 6 \mathrm{H}, J=6.18 \mathrm{~Hz}) .{ }^{13} \mathrm{C} \mathrm{NMR}\left(\mathrm{CDCl}_{3}, 100 \mathrm{MHz}\right) 159.1,136.5,130.8$, 130.2, 127.6, 126.1, 123.4, 79.4, 22.8. HRMS calculated for $\mathrm{C}_{10} \mathrm{H}_{13} \mathrm{NO}_{4} \mathrm{SBr}(\mathrm{M}+\mathrm{H})$ 321.9749; Found 321.9743.

\section{2-aminobenzenesulfonic acid (4)}<smiles>COS(=O)(=O)c1ccccc1NC=O</smiles>

Sulfonic ester 3a (100 mg, $0.47 \mathrm{mmol}$ ) was stirred in $\mathrm{MeOH}(8 \mathrm{~mL})$ and refluxed for $3 \mathrm{~h}$. Removal of solvent yielded complete conversion to product $4 .{ }^{1} \mathrm{H}$ NMR $\left(\mathrm{CD}_{3} \mathrm{OD}, 300 \mathrm{MHz}\right) \delta 7.82(\mathrm{~d}, 1 \mathrm{H}, J=7.8 \mathrm{~Hz}) 7.54(\mathrm{t}, 1 \mathrm{H}, J=7.7 \mathrm{~Hz})$ $7.45(\mathrm{t}, 1 \mathrm{H}, J=7.2 \mathrm{~Hz}) 7.35(\mathrm{~d}, 1 \mathrm{H}, J=7.9 \mathrm{~Hz}) .{ }^{13} \mathrm{C} \mathrm{NMR}\left(\mathrm{CD}_{3} \mathrm{OD}, 100 \mathrm{MHz}\right) \delta 138.1,135.8,131.7,131.0,130.5$, 126.9. Characterization data is consistent with authentic samples.

methyl 2-formamidobenzenesulfinate (5)<smiles></smiles>

This compound was synthesized following the General Procedure A with benzothiazole 1a (135 mg), $\mathrm{MeOH}(\mathbf{2 a})$ and a reaction time of $2 \mathrm{~h}$. This yielded 5 as a light yellow, flakey solid $(8.2 \mathrm{mg}, 0.04 \mathrm{mmol}, \mathbf{4 \%}$ yield). $\mathrm{Rf}=0.31(2: 3$ EtOAc/hexanes). ${ }^{1} \mathrm{H}$ NMR $\left(\mathrm{CDCl}_{3}, 300 \mathrm{MHz}\right.$ ) (Rotamer ratio: 4.2:1, Asterisk denotes minor peak) $\delta 10.11$ (br. s, $\left.1 \mathrm{H}\right)$ *9.00 (br. s, 1H) *8.75 (d, 1H, $J=10.81 \mathrm{~Hz}) 8.57(\mathrm{~d}, 1 \mathrm{H}, J=8.42 \mathrm{~Hz}) 8.46(\mathrm{~s}, 1 \mathrm{H}) * 7.66(\mathrm{~d}, 1 \mathrm{H}, J=7.47 \mathrm{~Hz}) 7.54$ $(\mathrm{t}, 1 \mathrm{H}, J=7.79 \mathrm{~Hz}) 7.45(\mathrm{~d}, 1 \mathrm{H}, J=7.75 \mathrm{~Hz}) * 7.35(\mathrm{~m}, 1 \mathrm{H}) 7.23(\mathrm{t}, 1 \mathrm{H}, J=7.59 \mathrm{~Hz}) 3.66(\mathrm{~s}, 3 \mathrm{H}) * 3.64(\mathrm{~s}, 3 \mathrm{H}) .{ }^{13} \mathrm{C}$ NMR $\left(\mathrm{CDCl}_{3}, 100 \mathrm{MHz}\right) \delta 159.6,138.4,133.7,128.1,127.6,124.3,122.9,51.6$. HRMS calculated for $\mathrm{C}_{8} \mathrm{H}_{9} \mathrm{NO}_{3} \mathrm{SLi}$ (M+Li) 206.0458; Found 206.0459. 


\section{Reaction Profile Data}

\section{Procedure for NMR kinetic Analysis}

A room temperature round bottom flask was charged with benzothiazole $(0.22 \mathrm{~mL}, 2.00 \mathrm{mmol}, 1.0$ equiv) and methanol $(10 \mathrm{~mL}, 0.2 \mathrm{M})$. Dodecane $(45 \mu \mathrm{L}, 0.2 \mathrm{mmol}, 0.1 \mathrm{eq})$ was then added to the reaction mixture. The reaction mixture was stirred vigorously at which point MMPP $(2.00 \mathrm{~g}, 3.20 \mathrm{mmol}, 1.6 \mathrm{eq})$ was added. After $5 \mathrm{~min}$, a $0.5 \mathrm{~mL}$ aliquot of the reaction mixture was removed, DI $\mathrm{H}_{2} \mathrm{O}(0.5 \mathrm{~mL})$ was added to the aliquot and the solution was extracted with $\mathrm{CH}_{2} \mathrm{Cl}_{2}(1 \mathrm{~mL}) 3$ times. The organic layers were collected, dried over $\mathrm{MgSO}_{4}$ and concentrated to yield the crude product. This process was repeated at the following times: 5min, $20 \mathrm{~min}, 1$ hour, 2 hours, 4 hours, 10 hours. After the data was collected, the relative concentrations of product and intermediate were determined by integrating $\delta 3.64$ (2) and $\delta 3.76$ (1) relative to $\delta 0.86$ (dodecane).

\section{Crystallographic Data}

\section{Product 3a single crystal $x$-ray structure}
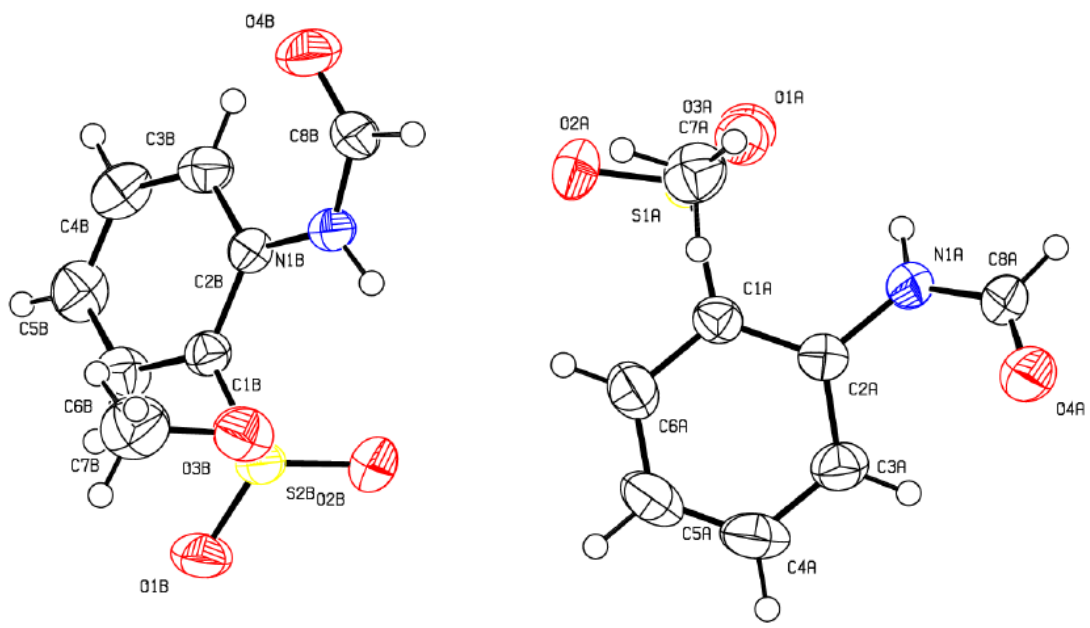

Table S1 - Crystal Data and Details of the Structure Determination

\begin{tabular}{|c|c|}
\hline \multicolumn{2}{|c|}{ Crystal Data } \\
\hline $\begin{array}{l}\text { Formula } \\
\mathrm{N} \mathrm{O4} \mathrm{S}\end{array}$ & $\mathrm{C} 8 \mathrm{H} 9$ \\
\hline $\begin{array}{l}\text { Formula Weight } \\
215.22\end{array}$ & \\
\hline $\begin{array}{l}\text { Crystal System } \\
\text { monoclinic }\end{array}$ & \\
\hline $\begin{array}{l}\text { Space group } \\
\text { (No. 14) }\end{array}$ & $\mathrm{P} 21 / \mathrm{c}$ \\
\hline $\begin{array}{l}\text { a, b, c [Angstrom] } \\
19.6081(6)\end{array}$ & $\begin{array}{ll}15.0399(4) & 8.2086(2)\end{array}$ \\
\hline 90 alpha, beta, gamma [deg] & $\begin{array}{ll}90 & 127.961(2)\end{array}$ \\
\hline
\end{tabular}




\begin{tabular}{|c|c|}
\hline $\begin{array}{l}\mathrm{V}[\text { Ang**3] } \\
1908.59(10)\end{array}$ & \\
\hline $8^{\mathrm{Z}}$ & \\
\hline & $\begin{array}{r}\mathrm{D} \text { (calc) }\left[\mathrm{g} / \mathrm{cm}^{* * 3]}\right. \\
1.498 \\
\end{array}$ \\
\hline $\begin{array}{l}\mathrm{Mu}(\mathrm{MoKa})[/ \mathrm{mm}] \\
0.327\end{array}$ & \\
\hline $8_{896}^{\mathrm{F}(000)}$ & \\
\hline 0.31 Crystal Size $[\mathrm{mm}]$ & $0.04 \times 0.12 \times$ \\
\hline Data Collec & \\
\hline 296 Temperature (K) & \\
\hline $\begin{array}{l}\text { Radiation [Angstrom] } \\
0.71073\end{array}$ & $\mathrm{MoKa}$ \\
\hline $\begin{array}{l}\text { Theta Min-Max [Deg] } \\
1.3,26.0\end{array}$ & \\
\hline $\begin{array}{l}\text { Dataset } \\
24: 23\end{array}$ & $-18: 18 ;-7: 10 ;-$ \\
\hline $\begin{array}{l}\text { Tot., Uniq. Data, } \mathrm{R}(\mathrm{int}) \\
0.021\end{array}$ & 18286,3749 \\
\hline $\begin{array}{l}\text { Observed Data [I > } 2.0 \text { sigma(I)] } \\
2920\end{array}$ & \\
\hline $\begin{array}{ll}\text { Refinemei } \\
\end{array}$ & \\
\hline $\begin{array}{l}\text { Nref, Npar } \\
3749,254\end{array}$ & \\
\hline $\begin{array}{l}\mathrm{R}, \mathrm{wR} 2, \mathrm{~S} \\
0.0743,1.17\end{array}$ & 0.0356 \\
\hline $\begin{array}{c}\mathrm{W}={ }^{\wedge} 2^{\wedge}\left(\mathrm{FO}^{\wedge} 2^{\wedge}\right)+(0.0139 \mathrm{P})^{\wedge} 2^{\wedge}+1.00 \\
\mathrm{P}=\left(\mathrm{FO}^{\wedge} 2^{\wedge}+2 \mathrm{FC}^{\wedge} 2^{\wedge}\right) / 3^{\prime}\end{array}$ & \\
\hline 0.00 Max. and Av. Shift/Error & 0.00 \\
\hline $\begin{array}{l}\text { Min. and Max. Resd. Dens. [e/Ang^3] } \\
0.30\end{array}$ & -0.30 \\
\hline
\end{tabular}

Table S2 - Final Coordinates and Equivalent Isotropic Displacement Parameters of the non-Hydrogen atoms

\begin{tabular}{|ccccc|}
\hline $\begin{array}{l}\text { Atom } \\
{[\text { Ang^2] }}\end{array}$ & $\mathrm{x}$ & $\mathrm{y}$ & $\mathrm{z}$ & $\mathrm{U}(\mathrm{eq})$ \\
\hline \multicolumn{6}{|c|}{} & & \\
\hline S1A & $0.71615(4)$ & $0.20387(6)$ & $0.67269(4)$ & $0.0430(2)$ \\
\hline S2B & $0.22022(4)$ & $0.04061(6)$ & $0.56054(3)$ & $0.0425(2)$ \\
\hline O1A & $0.76926(13)$ & $0.27082(18)$ & $0.63919(10)$ & $0.0548(6)$ \\
\hline \multicolumn{6}{c}{} \\
\hline
\end{tabular}




\begin{tabular}{|c|c|c|c|c|}
\hline $\mathrm{O} 2 \mathrm{~A}$ & $0.62224(13)$ & $0.28500(19)$ & $0.65777(11)$ & $0.0613(6)$ \\
\hline $\mathrm{O} 3 \mathrm{~A}$ & $0.81413(11)$ & $0.19298(17)$ & $0.77263(9)$ & $0.0465(5)$ \\
\hline $\mathrm{O} 4 \mathrm{~A}$ & $0.96367(13)$ & $-0.29009(19)$ & $0.75918(10)$ & $0.0594(6)$ \\
\hline N1A & $0.86732(13)$ & $-0.0612(2)$ & $0.68245(11)$ & $0.0410(6)$ \\
\hline $\mathrm{C} 1 \mathrm{~A}$ & $0.68004(16)$ & $-0.0005(2)$ & $0.64054(12)$ & $0.0383(6)$ \\
\hline O1B & $0.11610(12)$ & $-0.04113(18)$ & $0.50206(10)$ & $0.0610(5)$ \\
\hline $\mathrm{C} 2 \mathrm{~A}$ & $0.75687(16)$ & $-0.1103(2)$ & $0.64761(12)$ & $0.0372(6)$ \\
\hline $\mathrm{O} 2 \mathrm{~B}$ & $0.31904(12)$ & $-0.02296(19)$ & $0.57625(11)$ & $0.0612(6)$ \\
\hline $\mathrm{C} 3 \mathrm{~A}$ & $0.72127(18)$ & $-0.2687(3)$ & $0.61924(14)$ & $0.0492(8)$ \\
\hline $\mathrm{O} 3 \mathrm{~B}$ & $0.25126(12)$ & $0.04562(18)$ & $0.65265(9)$ & $0.0527(5)$ \\
\hline $\mathrm{C} 4 \mathrm{~A}$ & $0.6140(2)$ & $-0.3168(3) 0$ & & $0.0614(9)$ \\
\hline $\mathrm{O} 4 \mathrm{~B}$ & $0.46656(13)$ & $0.5549(2)$ & $0.72237(11)$ & $0.0741(6)$ \\
\hline $\mathrm{C} 5 \mathrm{~A}$ & $0.5397(2)$ & $-0.2090(3) 0$ & $0.58097(16)$ & $0.0637(9)$ \\
\hline C6A & $0.57206(18)$ & $-0.0500(3)$ & $0.60741(14)$ & $0.0520(8)$ \\
\hline C7A & $0.7845(2)$ & $0.1369(3) 0$ & $0.82650(16)$ & $0.0596(9)$ \\
\hline $\mathrm{C} 8 \mathrm{~A}$ & $0.96055(18)$ & $-0.1538(3)$ & $0.73429(14)$ & $0.0456(8)$ \\
\hline N1B & $0.39708(13)$ & $0.3115(2)$ & $0.65328(11)$ & $0.0465(6)$ \\
\hline $\mathrm{C} 1 \mathrm{~B}$ & $0.20335(16)$ & $0.2464(2)$ & $0.53025(12)$ & $0.0353(6)$ \\
\hline $\mathrm{C} 2 \mathrm{~B}$ & $0.29273(16)$ & $0.3582(2)$ & $0.57712(12)$ & $0.0354(6)$ \\
\hline $\mathrm{C} 3 \mathrm{~B}$ & $0.27208(18)$ & $0.5171(3)$ & $0.54549(14)$ & $0.0438(7)$ \\
\hline C4B & $0.1671(2)$ & $0.5623(3) 0$ & $.47267(14)$ & $0.0490(8)$ \\
\hline
\end{tabular}




\begin{tabular}{|lllll|}
\hline \multicolumn{5}{|c|}{} \\
\hline C5B & $0.08012(19)$ & $0.4528(3)$ & $0.42895(14)$ & $0.0522(8)$ \\
\hline C6B & $0.09841(17)$ & $0.2947(3)$ & $0.45719(13)$ & $0.0452(7)$ \\
\hline \multicolumn{5}{c}{} \\
\hline C7B & $0.1670(2)$ & $0.1025(3)$ & $0.66047(18)$ & $0.0709(11)$ \\
\hline C8B & $0.47454(17)$ & $0.4096(3)$ & $0.71859(15)$ & $0.0539(8)$ \\
\hline
\end{tabular}

Table S3 - Hydrogen Atom Positions and Isotropic Displacement Parameters

\begin{tabular}{|c|c|c|c|c|}
\hline $\begin{array}{l}\text { Atom } \\
{[\text { [Ang^2] }}\end{array}$ & $\mathrm{x}$ & $\mathrm{y}$ & $\mathrm{Z}$ & U(iso) \\
\hline H1AA & 0.87580 & 0.03500 & 0.66990 & 0.0490 \\
\hline H3AA & 0.77000 & -0.34310 & 0.62200 & 0.0590 \\
\hline H4AA & 0.59160 & -0.42390 & 0.56880 & 0.0740 \\
\hline H5AA & 0.46770 & -0.24320 & 0.55920 & 0.0760 \\
\hline H6AA & 0.52180 & 0.02360 & 0.60300 & 0.0620 \\
\hline H7AA & 0.85070 & 0.13530 & 0.88590 & 0.0720 \\
\hline H7AB & 0.72960 & 0.20920 & 0.82010 & 0.0720 \\
\hline H7AC & 0.75350 & 0.02900 & 0.80910 & 0.0720 \\
\hline H8AA & 1.02870 & -0.10860 & 0.75260 & 0.0550 \\
\hline H1BA & 0.41320 & 0.20950 & 0.65890 & 0.0560 \\
\hline H3BA & 0.33000 & 0.59340 & 0.57390 & 0.0530 \\
\hline H4BA & 0.15510 & 0.66910 & 0.45290 & 0.0590 \\
\hline H5BA & 0.00920 & 0.48540 & 0.38050 & 0.0630 \\
\hline H6BA & 0.03980 & 0.21960 & 0.42730 & 0.0540 \\
\hline
\end{tabular}




\begin{tabular}{|ccccc|}
\hline H7BA & 0.19710 & 0.09930 & 0.72010 & 0.0850 \\
\hline & & & & \\
\hline H7BB & 0.14630 & 0.21240 & 0.63950 & 0.0850 \\
\hline H7BC & 0.10170 & 0.03380 & 0.62700 & 0.0850 \\
\hline & & & & \\
\hline H8BA & 0.54050 & 0.36070 & 0.76520 & 0.0650 \\
\hline
\end{tabular}

Table S4 - Bond Distances (Angstrom)

\begin{tabular}{|c|c|}
\hline $\begin{array}{rr}\text { S1A } & -01 \mathrm{~A} \\
0.9300 & \\
\end{array}$ & $1.420(2) \quad \mathrm{C} 3 \mathrm{~A} \quad-\mathrm{H} 3 \mathrm{AA}$ \\
\hline $\begin{aligned} \text { S1A } & -\mathrm{O} 2 \mathrm{~A} \\
0.9300 & \end{aligned}$ & C4A - -H4AA \\
\hline $\begin{array}{ll}\text { S1A } & -\mathrm{O} 3 \mathrm{~A} \\
0.9300 & \\
\end{array}$ & $1.5707(15) \quad$ C5A $\quad-\mathrm{H} 5 \mathrm{AA}$ \\
\hline $\begin{array}{ll}\text { S1A } & -\mathrm{C} 1 \mathrm{~A} \\
0.9300 & \\
\end{array}$ & 1.7566(17) C6A $\quad$-H6AA \\
\hline $\begin{array}{ll}\text { S2B } & -\mathrm{C} 1 \mathrm{~B} \\
0.9600 & \end{array}$ & $1.7565(17) \quad$ C7A $\quad-$ H7AA \\
\hline $\begin{aligned} \text { S2B } & -\mathrm{O} 1 \mathrm{~B} \\
0.9600 & \end{aligned}$ & 1.4171(19) $\quad$ C7A $\quad-$ H7AC \\
\hline $\begin{array}{ll}\text { S2B } & -\mathrm{O} 2 \mathrm{~B} \\
0.9600 & \end{array}$ & 1.419(2) $\quad$ C7A $\quad-\mathrm{H} 7 \mathrm{AB}$ \\
\hline $\begin{array}{ll}\text { S2B } & -\mathrm{O} 3 \mathrm{~B} \\
0.9300 & \end{array}$ & $1.5634(16) \quad$ C8A $\quad-H 8 A A$ \\
\hline $\begin{array}{rr}\text { O3A } & -\mathrm{C} 7 \mathrm{~A} \\
1.405(3) & \\
\end{array}$ & 1.451(4) $\quad$ C1B $\quad-$ C2B \\
\hline $\begin{array}{rr}\mathrm{O} 4 \mathrm{~A} & -\mathrm{C} 8 \mathrm{~A} \\
1.385(3) & \\
\end{array}$ & 1.210(3) $\quad$ C1B $\quad-$-C6B \\
\hline $\begin{array}{rr}\mathrm{N} 1 \mathrm{~A} & -\mathrm{C} 2 \mathrm{~A} \\
0.8600 & \\
\end{array}$ & 1.412(3) $\quad \mathrm{N} 1 \mathrm{~B} \quad-\mathrm{H} 1 \mathrm{BA}$ \\
\hline $\begin{array}{rr}\mathrm{N} 1 \mathrm{~A} & -\mathrm{C} 8 \mathrm{~A} \\
1.395(3) & \\
\end{array}$ & $\begin{array}{lll}1.350(3) & \mathrm{C} 2 \mathrm{~B} & -\mathrm{C} 3 \mathrm{~B}\end{array}$ \\
\hline
\end{tabular}




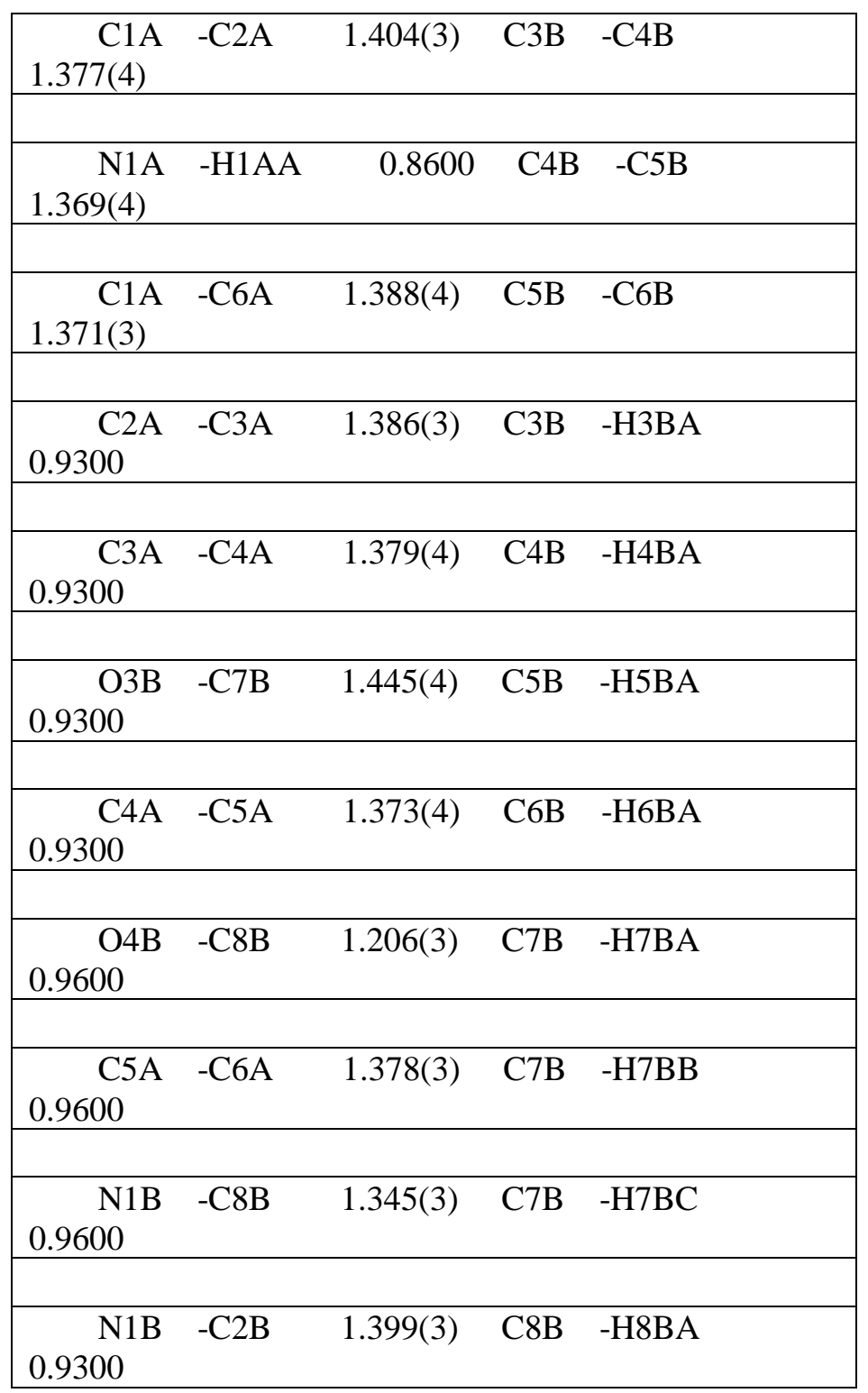

Table S5 - Bond Angles (Degrees)

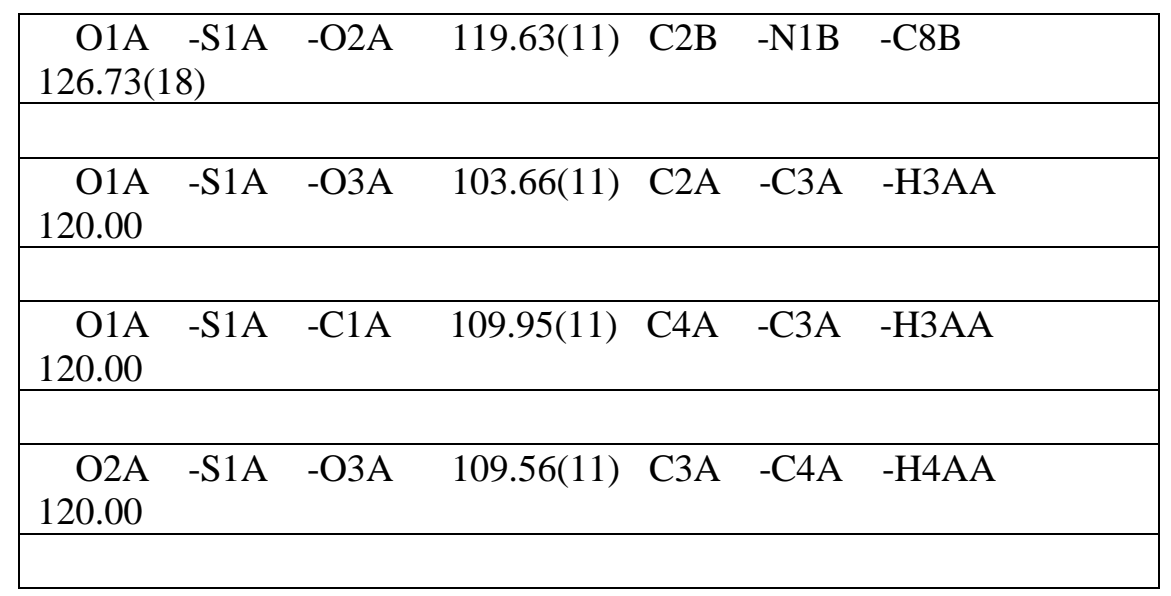




\begin{tabular}{|c|c|c|c|}
\hline $\begin{array}{r}\mathrm{O} 2 \mathrm{~A} \\
119.00\end{array}$ & $-\mathrm{S} 1 \mathrm{~A}$ & $-\mathrm{C} 1 \mathrm{~A}$ & 108.98(12) C5A $-\mathrm{C} 4 \mathrm{~A} \quad-\mathrm{H} 4 \mathrm{AA}$ \\
\hline $\begin{array}{r}\text { O3A } \\
120.00 \\
\end{array}$ & $-\mathrm{S} 1 \mathrm{~A}$ & $-\mathrm{C} 1 \mathrm{~A}$ & 103.83(8) C4A $\quad-$ C5A \\
\hline $\begin{array}{r}\text { O1B } \\
120.00 \\
\end{array}$ & $-\mathrm{S} 2 \mathrm{~B}$ & $-\mathrm{O} 2 \mathrm{~B}$ & 119.35(11) C6A $\quad-\mathrm{C} 5 \mathrm{~A} \quad-\mathrm{H} 5 \mathrm{AA}$ \\
\hline $\begin{array}{r}\text { O1B } \\
120.00\end{array}$ & $-\mathrm{S} 2 \mathrm{~B}$ & $-\mathrm{O} 3 \mathrm{~B}$ & 110.08(11) C1A $\quad$-C6A $\quad$-H6AA \\
\hline $\begin{array}{r}\text { O1B } \\
120.00\end{array}$ & $-\mathrm{S} 2 \mathrm{~B}$ & $-\mathrm{C} 1 \mathrm{~B}$ & 108.90(10) $\quad$ C5A $\quad-$ - 6 A $\quad-$-H6AA \\
\hline $\begin{array}{r}\mathrm{O} 2 \mathrm{~B} \\
109.00 \\
\end{array}$ & $-\mathrm{S} 2 \mathrm{~B}$ & $-\mathrm{O} 3 \mathrm{~B}$ & 103.32(10) O3A $\quad-\mathrm{C} 7 \mathrm{~A} \quad-\mathrm{H} 7 \mathrm{AB}$ \\
\hline $\begin{array}{r}\mathrm{O} 2 \mathrm{~B} \\
109.00 \\
\end{array}$ & $-\mathrm{S} 2 \mathrm{~B}$ & $-\mathrm{C} 1 \mathrm{~B}$ & 109.96(12) O3A - -C7A $-\mathrm{H} 7 \mathrm{AC}$ \\
\hline $\begin{array}{r}\text { O3B } \\
109.00\end{array}$ & $-\mathrm{S} 2 \mathrm{~B}$ & $-\mathrm{C} 1 \mathrm{~B}$ & 104.11(9) H7AA -C7A -H7AB \\
\hline $\begin{array}{r}\text { S1A } \\
109.00 \\
\end{array}$ & $-\mathrm{O} 3 \mathrm{~A}$ & $-\mathrm{C} 7 \mathrm{~A}$ & 116.98(17) H7AA -C7A - -H7AC \\
\hline $\begin{array}{r}\mathrm{C} 2 \mathrm{~A} \\
109.00 \\
\end{array}$ & $-\mathrm{N} 1 \mathrm{~A}$ & $-\mathrm{C} 8 \mathrm{~A}$ & 124.86(19) $\quad$ H7AB $\quad-$ C7A $\quad-H 7 A C$ \\
\hline $\begin{array}{r}\text { S1A } \\
110.00\end{array}$ & $-\mathrm{C} 1 \mathrm{~A}$ & $-\mathrm{C} 2 \mathrm{~A}$ & 121.59(19) $\quad$ O3A $\quad-$ C7A $\quad-$ H7AA \\
\hline $\begin{array}{r}\mathrm{C} 2 \mathrm{~A} \\
117.00 \\
\end{array}$ & $-\mathrm{N} 1 \mathrm{~A}$ & -H1AA & $117.00 \quad 04 \mathrm{~A} \quad-\mathrm{C} 8 \mathrm{~A} \quad-\mathrm{H} 8 \mathrm{AA}$ \\
\hline $\begin{array}{r}\text { C8A } \\
117.00 \\
\end{array}$ & $-\mathrm{N} 1 \mathrm{~A}$ & -H1AA & $118.00 \quad \mathrm{~N} 1 \mathrm{~A} \quad-\mathrm{C} 8 \mathrm{~A} \quad-\mathrm{H} 8 \mathrm{AA}$ \\
\hline $\begin{array}{c}\text { S1A } \\
121.73(1 \\
\end{array}$ & $\begin{array}{l}-\mathrm{C} 1 \mathrm{~A} \\
5)\end{array}$ & $-\mathrm{C6A}$ & $117.52(18) \quad$ S2B $\quad-C 1 B \quad-C 2 B$ \\
\hline $\begin{array}{c}\mathrm{C} 2 \mathrm{~A} \\
117.43(1\end{array}$ & $\begin{array}{l}-\mathrm{C} 1 \mathrm{~A} \\
7) \\
\end{array}$ & $-\mathrm{C} 6 \mathrm{~A}$ & 120.89(18) $\quad$ S2B $\quad-$ C1B $\quad-$ C6B \\
\hline $\begin{array}{c}\mathrm{C} 1 \mathrm{~A} \\
120.83(1 \\
\end{array}$ & $\begin{array}{l}-\mathrm{C} 2 \mathrm{~A} \\
8)\end{array}$ & $-\mathrm{C} 3 \mathrm{~A}$ & 118.1(2) $\quad$ C2B $\quad-$ C1B $\quad-$ C6B \\
\hline
\end{tabular}




\begin{tabular}{|c|c|c|}
\hline $\begin{aligned} & \mathrm{N} 1 \mathrm{~A}-\mathrm{C} 2 \mathrm{~A} \\
& 117.00 \\
&\end{aligned}$ & $-\mathrm{C} 3 \mathrm{~A}$ & $\begin{array}{llll}120.8(2) & \text { C2B } & -\mathrm{N} 1 \mathrm{~B} & -\mathrm{H} 1 \mathrm{BA} \\
\end{array}$ \\
\hline $\begin{array}{rr}\mathrm{N} 1 \mathrm{~A} & -\mathrm{C} 2 \mathrm{~A} \\
117.00 & \\
\end{array}$ & $-\mathrm{C} 1 \mathrm{~A}$ & 121.11(16) $\mathrm{C} 8 \mathrm{~B} \quad-\mathrm{N} 1 \mathrm{~B} \quad-\mathrm{H} 1 \mathrm{BA}$ \\
\hline $\begin{array}{l}\mathrm{C} 2 \mathrm{~A}-\mathrm{C} 3 \mathrm{~A} \\
120.87(16)\end{array}$ & $-\mathrm{C} 4 \mathrm{~A}$ & $\begin{array}{llll}120.5(3) & \text { N1B } & -\mathrm{C} 2 \mathrm{~B} & -\mathrm{C} 1 \mathrm{~B} \\
\end{array}$ \\
\hline $\begin{array}{ll}\mathrm{S} 2 \mathrm{~B} & -\mathrm{O} 3 \mathrm{~B} \\
117.3(2) & \end{array}$ & $-\mathrm{C} 7 \mathrm{~B}$ & 118.24(15) $\quad$ C1B $\quad-$ C2B $\quad-C 3 B$ \\
\hline $\begin{array}{c}\text { C3A } \\
121.9(2)\end{array}$ & $-\mathrm{C} 5 \mathrm{~A}$ & $\begin{array}{llll}121.0(2) & \text { N1B } & -\mathrm{C} 2 \mathrm{~B} & -\mathrm{C} 3 \mathrm{~B}\end{array}$ \\
\hline $\begin{array}{cc}\mathrm{C} 4 \mathrm{~A} & -\mathrm{C} 5 \mathrm{~A} \\
120.9(2) & \end{array}$ & $-\mathrm{C} 6 \mathrm{~A}$ & $\begin{array}{llll}119.9(3) & \mathrm{C} 2 \mathrm{~B} & -\mathrm{C} 3 \mathrm{~B} & -\mathrm{C} 4 \mathrm{~B}\end{array}$ \\
\hline $\begin{array}{ll}\text { C1A } & -\mathrm{C} 6 \mathrm{~A} \\
121.1(2) & \\
\end{array}$ & $-\mathrm{C} 5 \mathrm{~A}$ & 119.6(3) $\quad$ C3B $\quad-$-C4B $\quad-$-C5B \\
\hline $\begin{array}{cc}\text { O4A } & -\mathrm{C} 8 \mathrm{~A} \\
119.4(2) & \\
\end{array}$ & $-\mathrm{N} 1 \mathrm{~A}$ & 126.1(3) $\mathrm{C} 4 \mathrm{~B} \quad-\mathrm{C} 5 \mathrm{~B} \quad-\mathrm{C} 6 \mathrm{~B}$ \\
\hline $\begin{aligned} \mathrm{C} 1 \mathrm{~B} & -\mathrm{C} 6 \mathrm{~B} \\
120.00 & \end{aligned}$ & $-\mathrm{C} 5 \mathrm{~B}$ & 120.4(2) $\mathrm{C} 5 \mathrm{~B} \quad$-C6B $\quad$-H6BA \\
\hline $\begin{array}{rr}\mathrm{O} 4 \mathrm{~B} & -\mathrm{C} 8 \mathrm{~B} \\
109.00 & \\
\end{array}$ & $-\mathrm{N} 1 \mathrm{~B}$ & 126.2(2) O3B $\quad-\mathrm{C} 7 \mathrm{~B} \quad-\mathrm{H} 7 \mathrm{BA}$ \\
\hline $\begin{array}{rr}\mathrm{C} 2 \mathrm{~B} & -\mathrm{C} 3 \mathrm{~B} \\
109.00 & \\
\end{array}$ & $-\mathrm{H} 3 \mathrm{BA}$ & $\begin{array}{llll}120.00 & \text { O3B } & -\mathrm{C} 7 \mathrm{~B} & -\mathrm{H} 7 \mathrm{BB}\end{array}$ \\
\hline $\begin{array}{rr}\mathrm{C} 4 \mathrm{~B} & -\mathrm{C} 3 \mathrm{~B} \\
109.00 & \\
\end{array}$ & $-\mathrm{H} 3 \mathrm{BA}$ & $120.00 \quad$ O3B $\quad-$ C7B $\quad-H 7 B C$ \\
\hline $\begin{aligned} \mathrm{C} 3 \mathrm{~B} & -\mathrm{C} 4 \mathrm{~B} \\
109.00 & \end{aligned}$ & $-\mathrm{H} 4 \mathrm{BA}$ & $119.00 \quad$ H7BA $\quad$-C7B $\quad$-H7BB \\
\hline $\begin{aligned} & \text { C5B }-\mathrm{C} 4 \mathrm{~B} \\
& 110.00 \\
&\end{aligned}$ & $-\mathrm{H} 4 \mathrm{BA}$ & $119.00 \quad \mathrm{H} 7 \mathrm{BA} \quad-\mathrm{C} 7 \mathrm{~B} \quad-\mathrm{H} 7 \mathrm{BC}$ \\
\hline $\begin{array}{rr}\mathrm{C} 4 \mathrm{~B} & -\mathrm{C} 5 \mathrm{~B} \\
109.00 & \\
\end{array}$ & -H5BA & $120.00 \quad \mathrm{H} 7 \mathrm{BB} \quad-\mathrm{C} 7 \mathrm{~B} \quad-\mathrm{H} 7 \mathrm{BC}$ \\
\hline
\end{tabular}




\begin{tabular}{|rllllll|}
\hline C6B & $-\mathrm{C} 5 \mathrm{~B}$ & $-\mathrm{H} 5 \mathrm{BA}$ & 120.00 & O4B & $-\mathrm{C} 8 \mathrm{~B}$ & $-\mathrm{H} 8 \mathrm{BA}$ \\
117.00 & & & & & & \\
\hline & & & & & & \\
\hline $\mathrm{C} 1 \mathrm{~B}$ & $-\mathrm{C} 6 \mathrm{~B}$ & $-\mathrm{H} 6 \mathrm{BA}$ & 120.00 & $\mathrm{~N} 1 \mathrm{~B}$ & $-\mathrm{C} 8 \mathrm{~B}$ & $-\mathrm{H} 8 \mathrm{BA}$ \\
117.00 & & & & & & \\
\hline
\end{tabular}

\section{Product 4 twin crystal $x-$ ray data}

Single crystal x-ray structure model incomplete due to weak data.
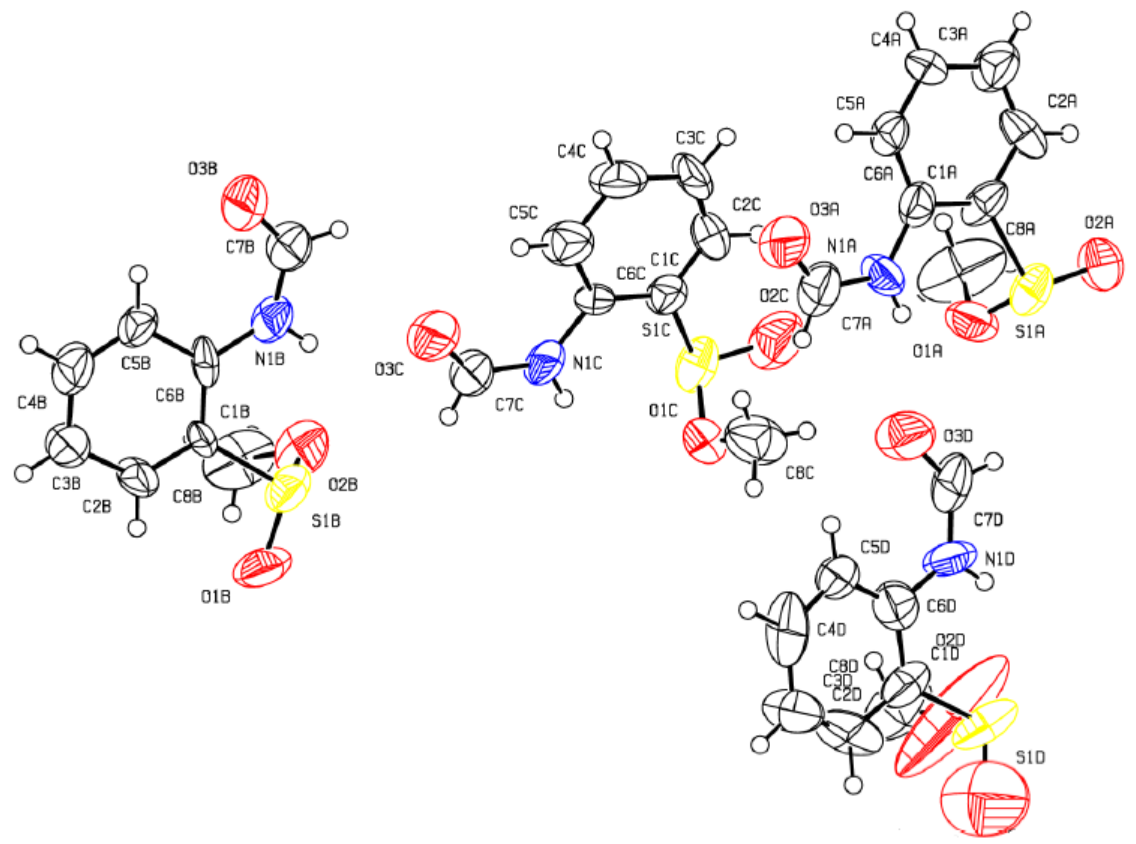
4. Spectral Data of New Compounds

$$
\text { iH NMR Spectrum }
$$

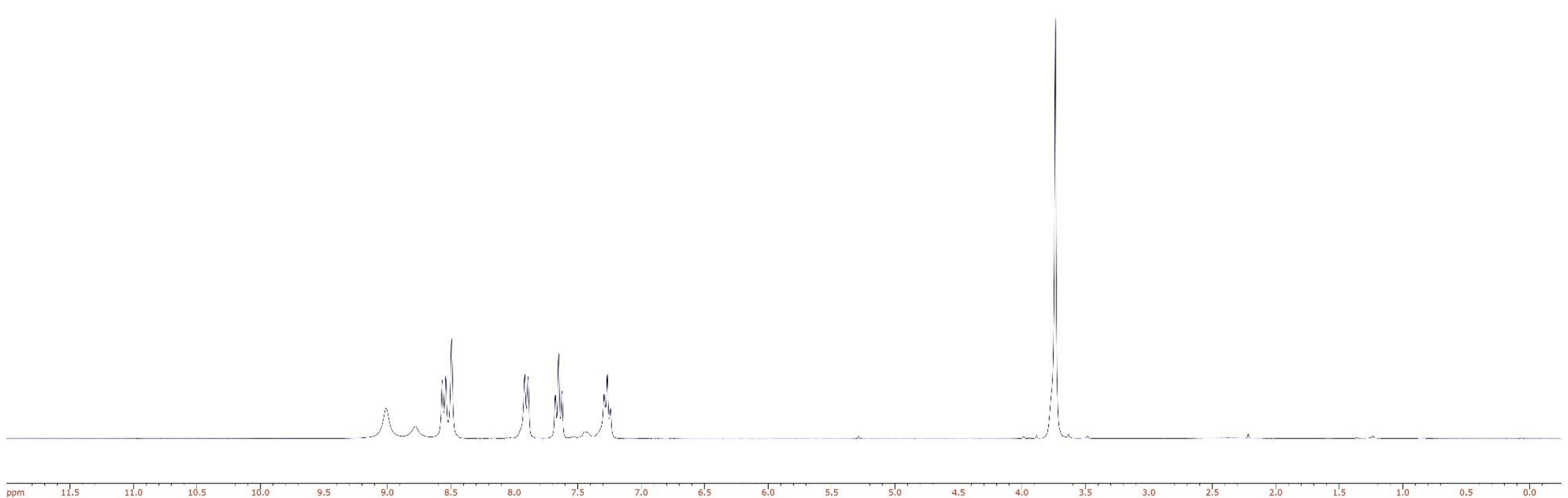




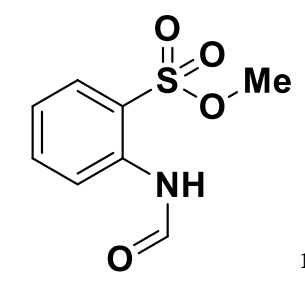

${ }^{13}$ C NMR Spectrum

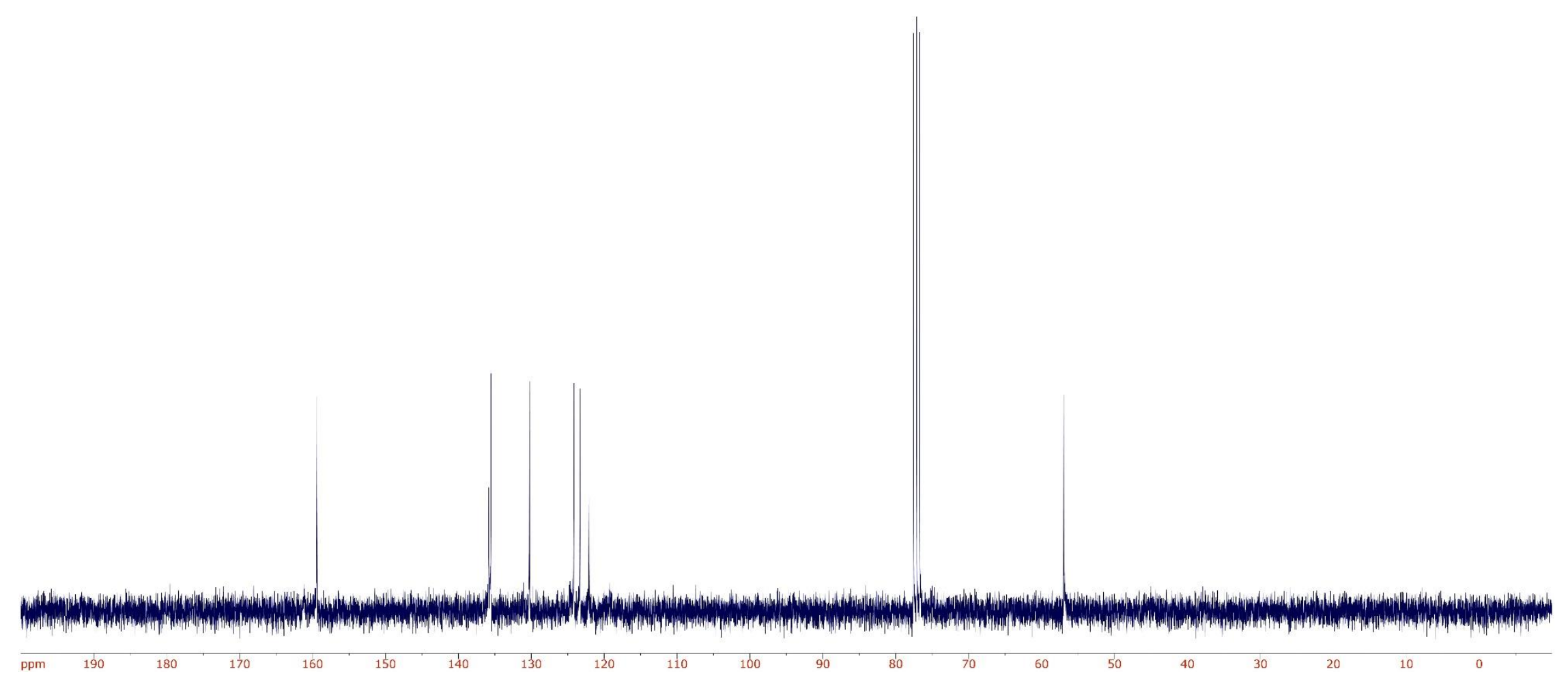




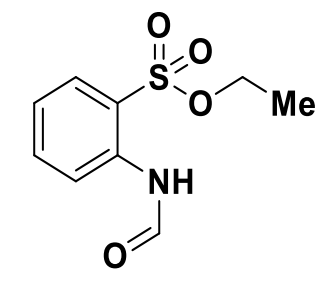

${ }^{1}$ H NMR Spectrum

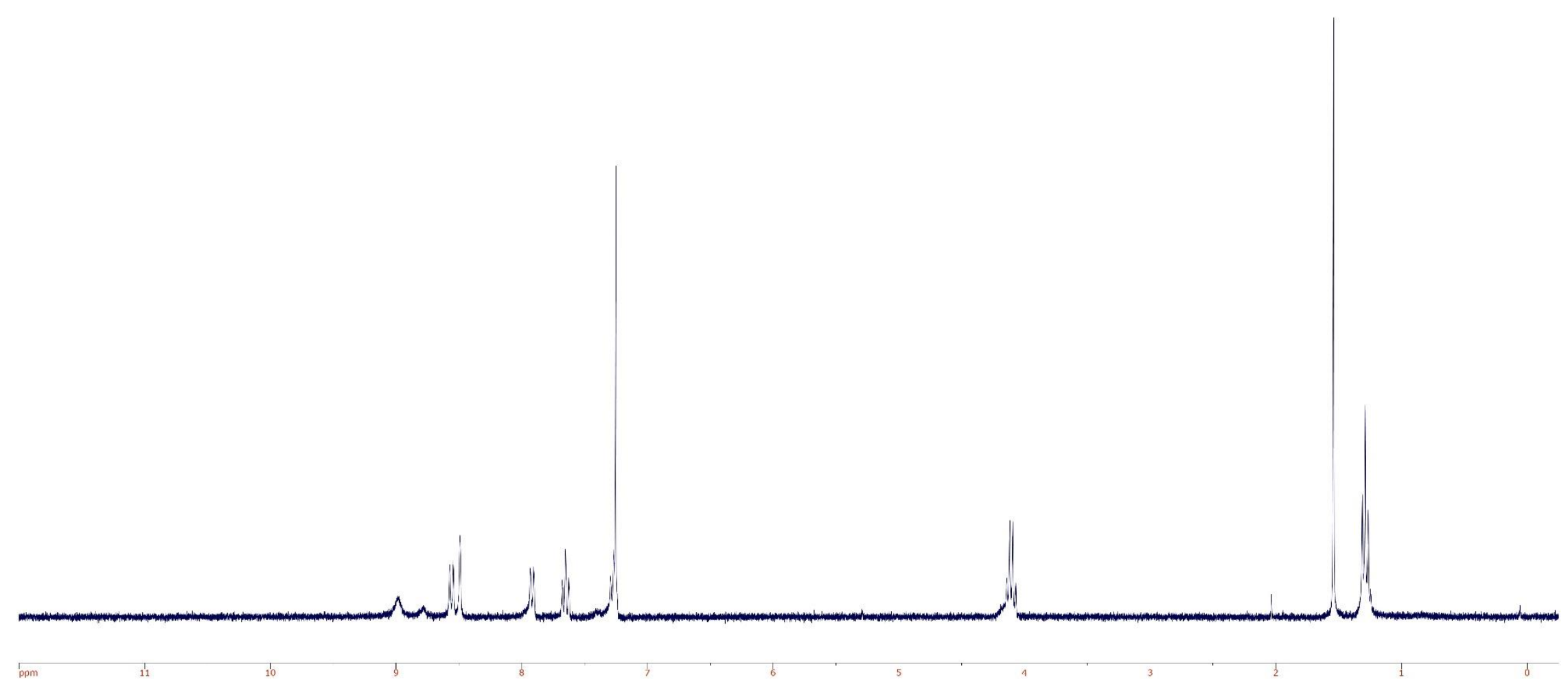




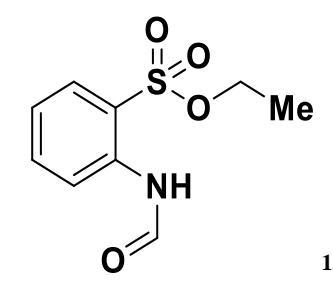

${ }^{13}$ C NMR Spectrum

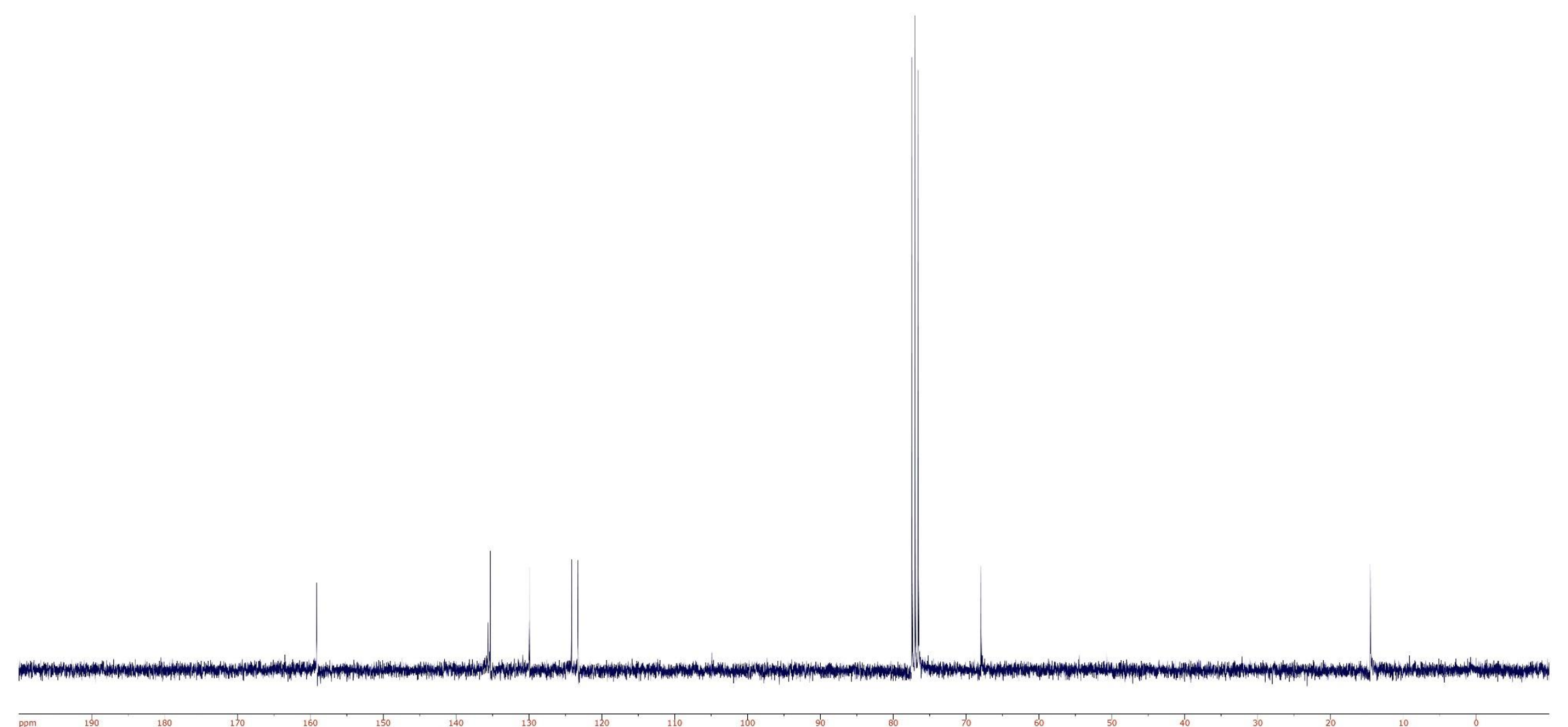




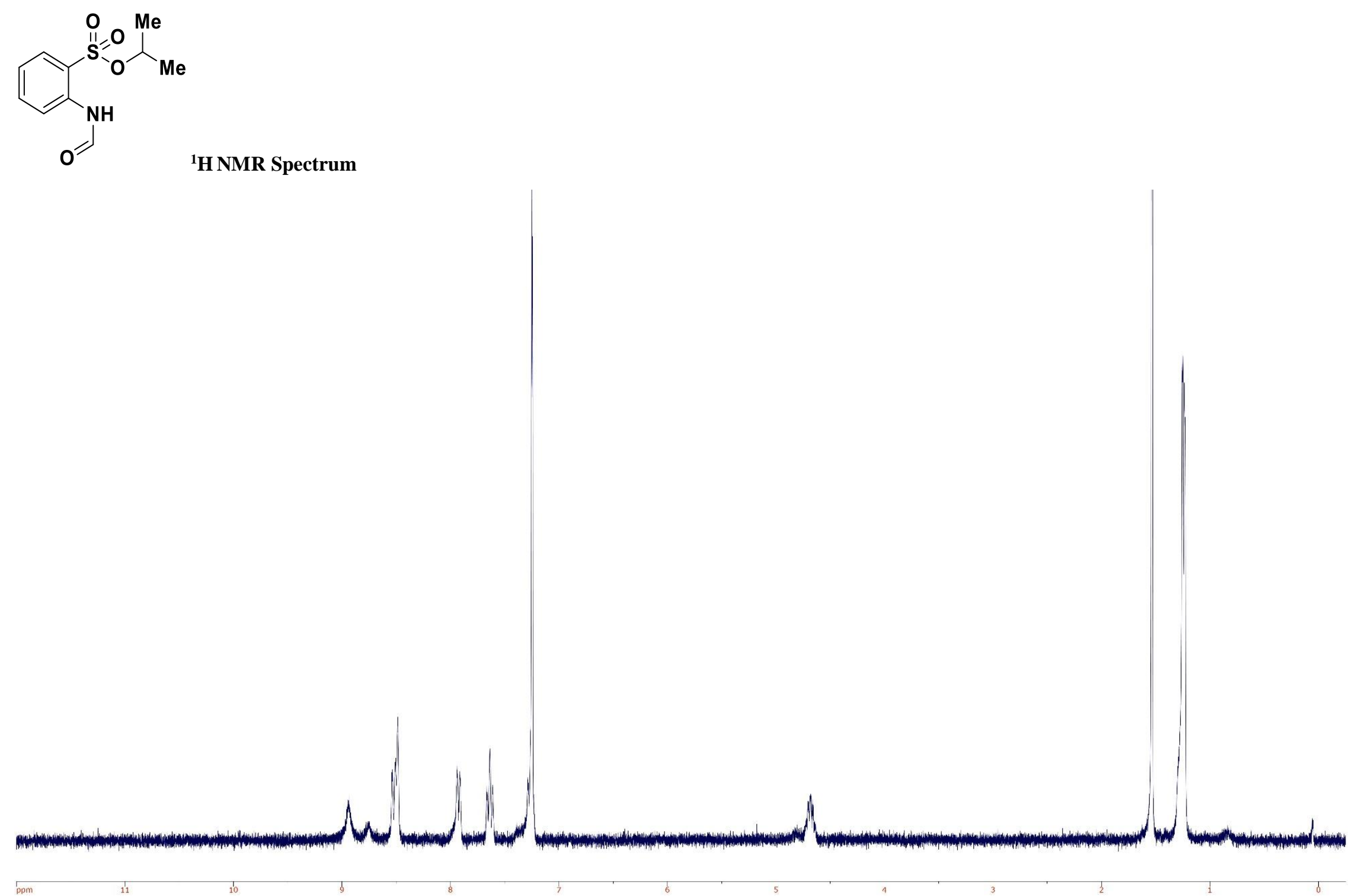




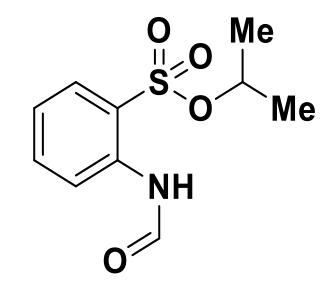

${ }^{13}$ C NMR Spectrum

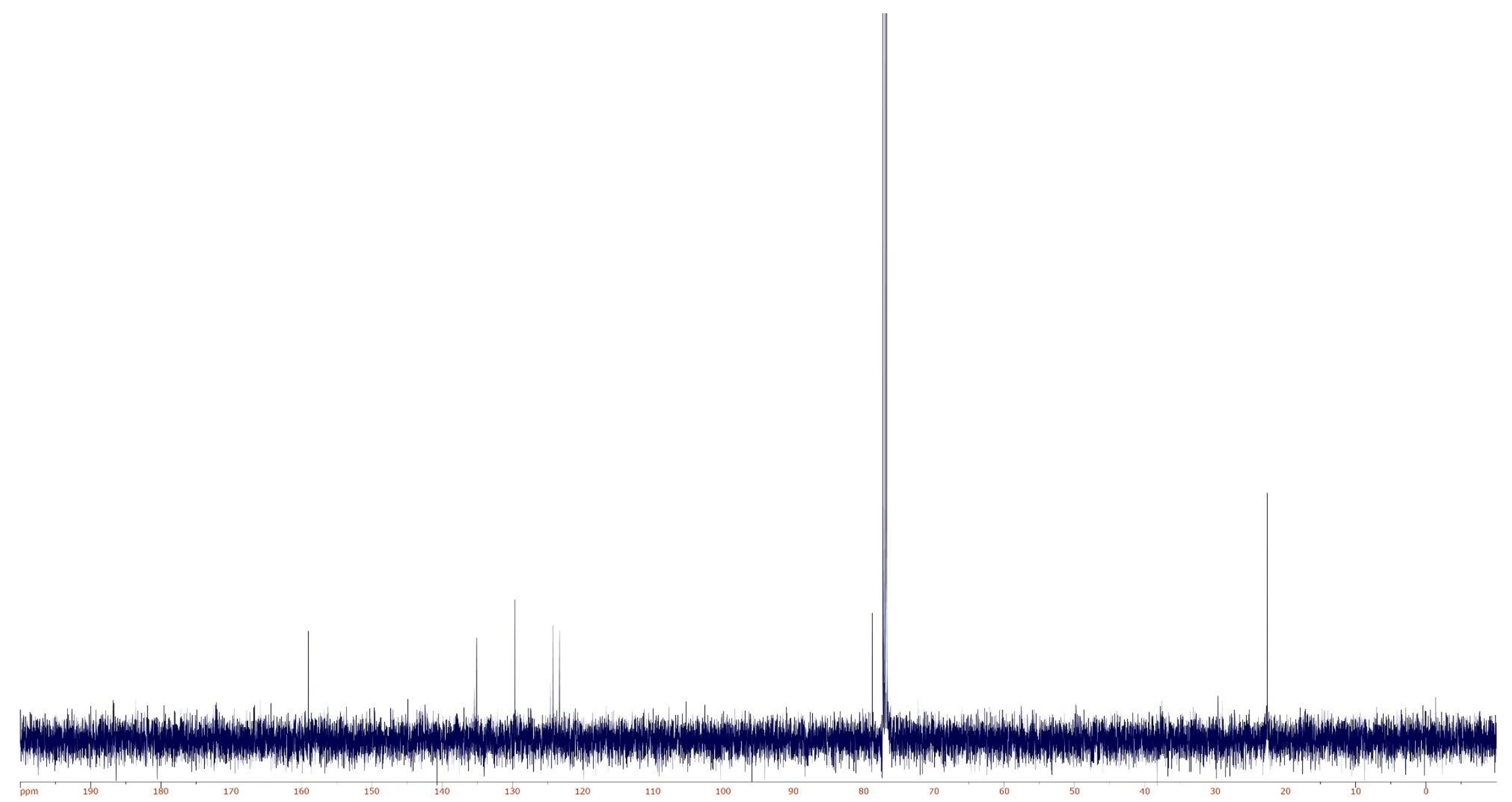




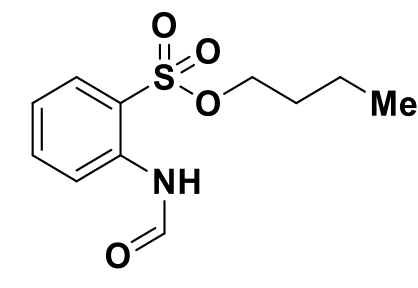

${ }^{1}$ H NMR Spectrum

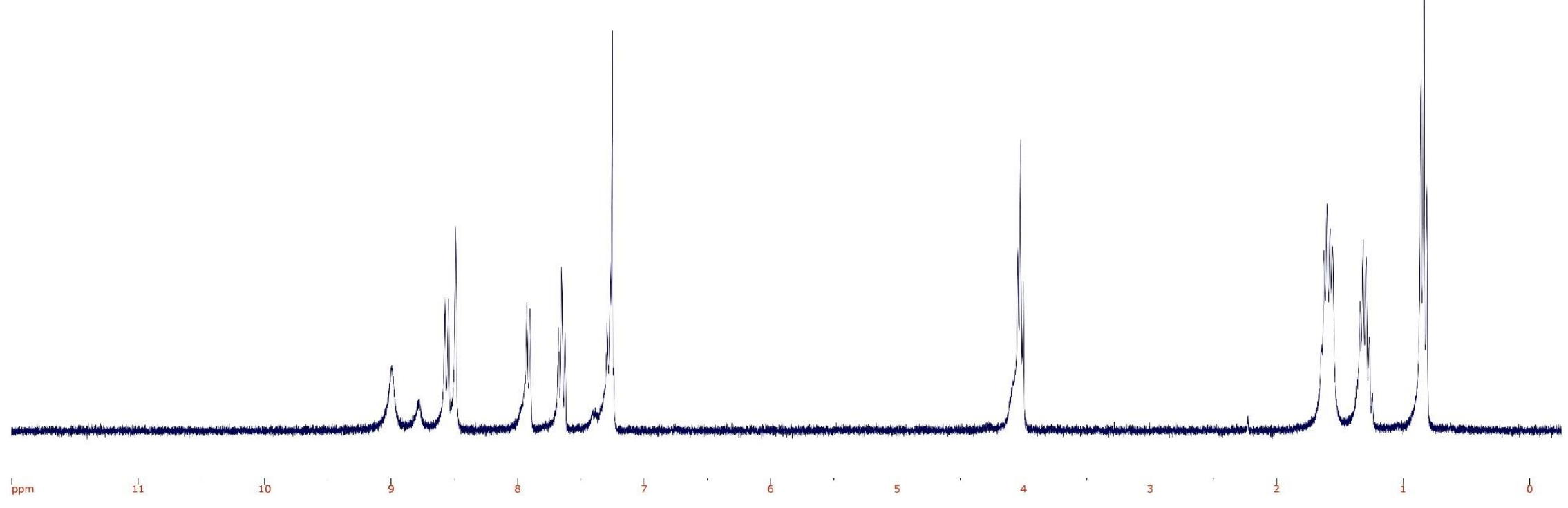




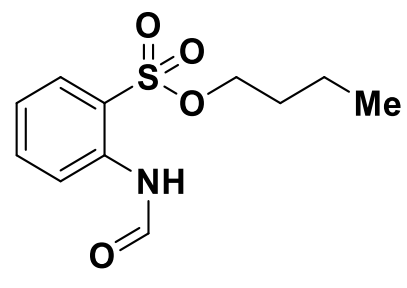

${ }^{13}$ C NMR Spectrum

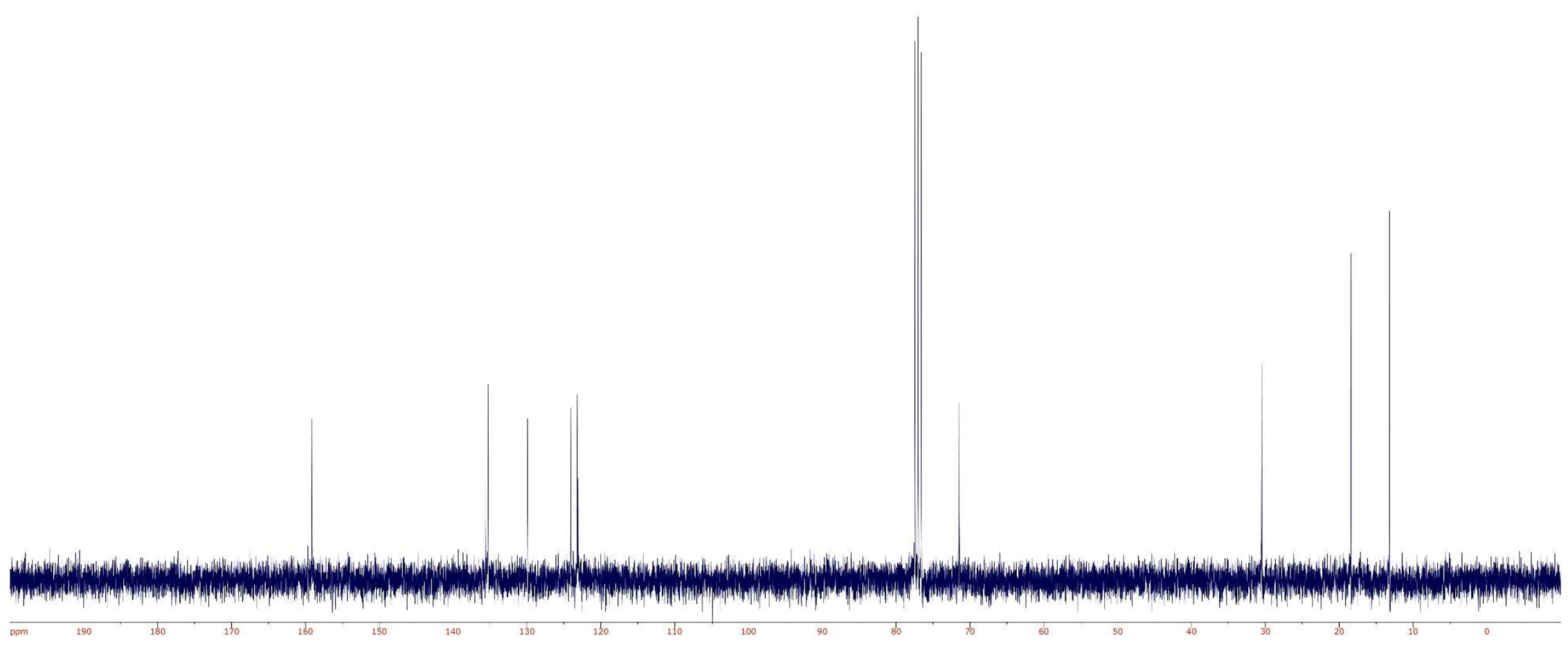




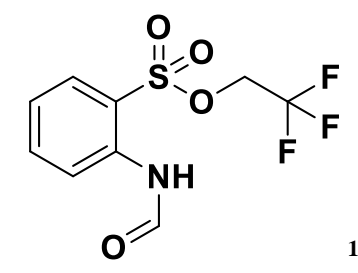

${ }^{1}$ H NMR Spectrum

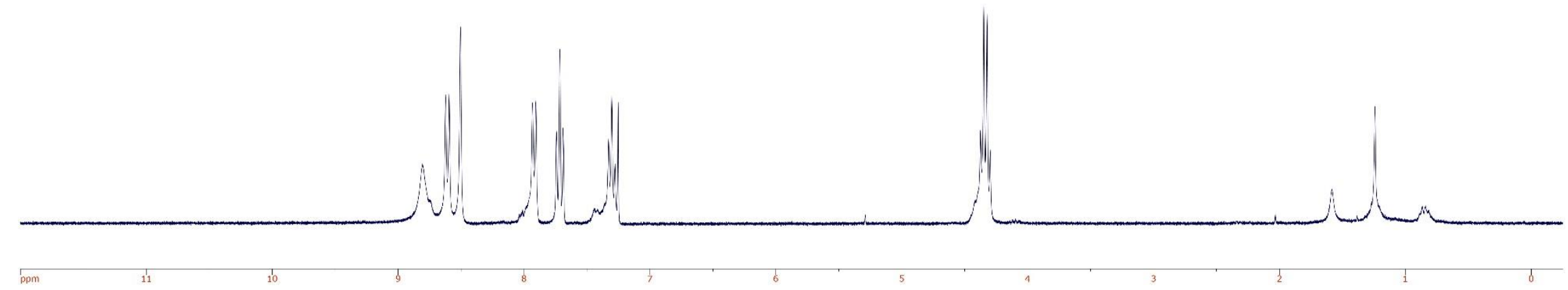




$$
\begin{aligned}
& \overbrace{N H}^{\stackrel{O}{11}=0}=\overbrace{F}^{F} \\
& 0=
\end{aligned}
$$

${ }^{13}$ C NMR Spectrum

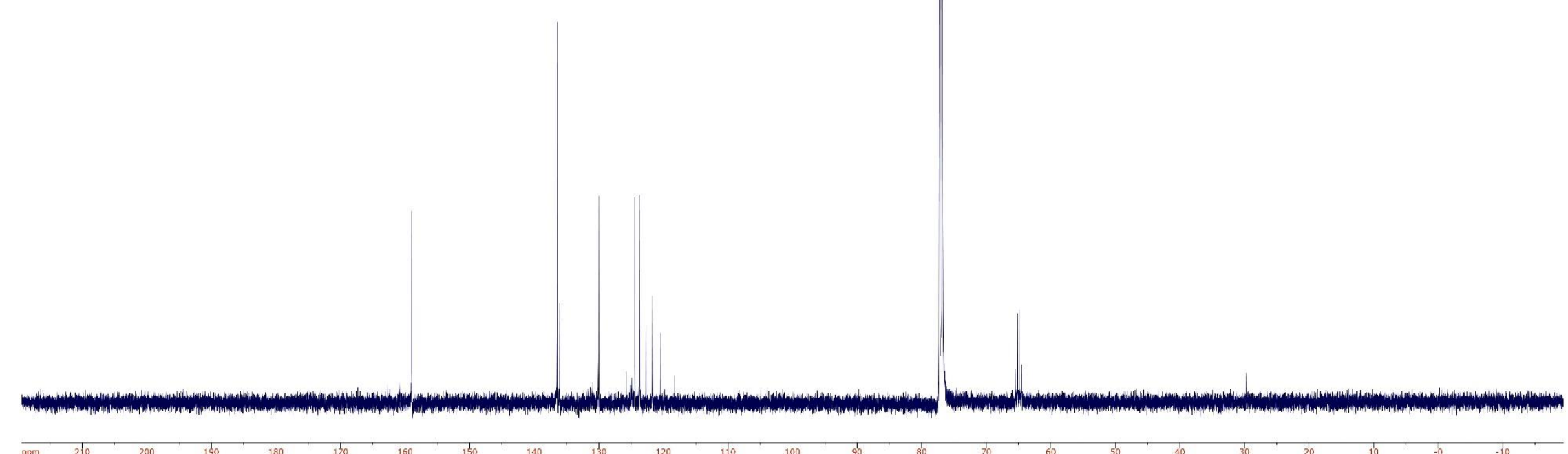




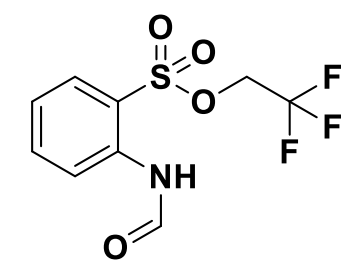

${ }^{19}$ F NMR Spectrum 


$$
\begin{aligned}
& \overbrace{\mathrm{NH}}^{\mathrm{O}} \mathrm{N}_{\mathrm{O}}^{\mathrm{II}=0} \\
& { }_{\mathrm{O}}{ }^{1} \mathrm{H} \text { NMR Spectrum }
\end{aligned}
$$

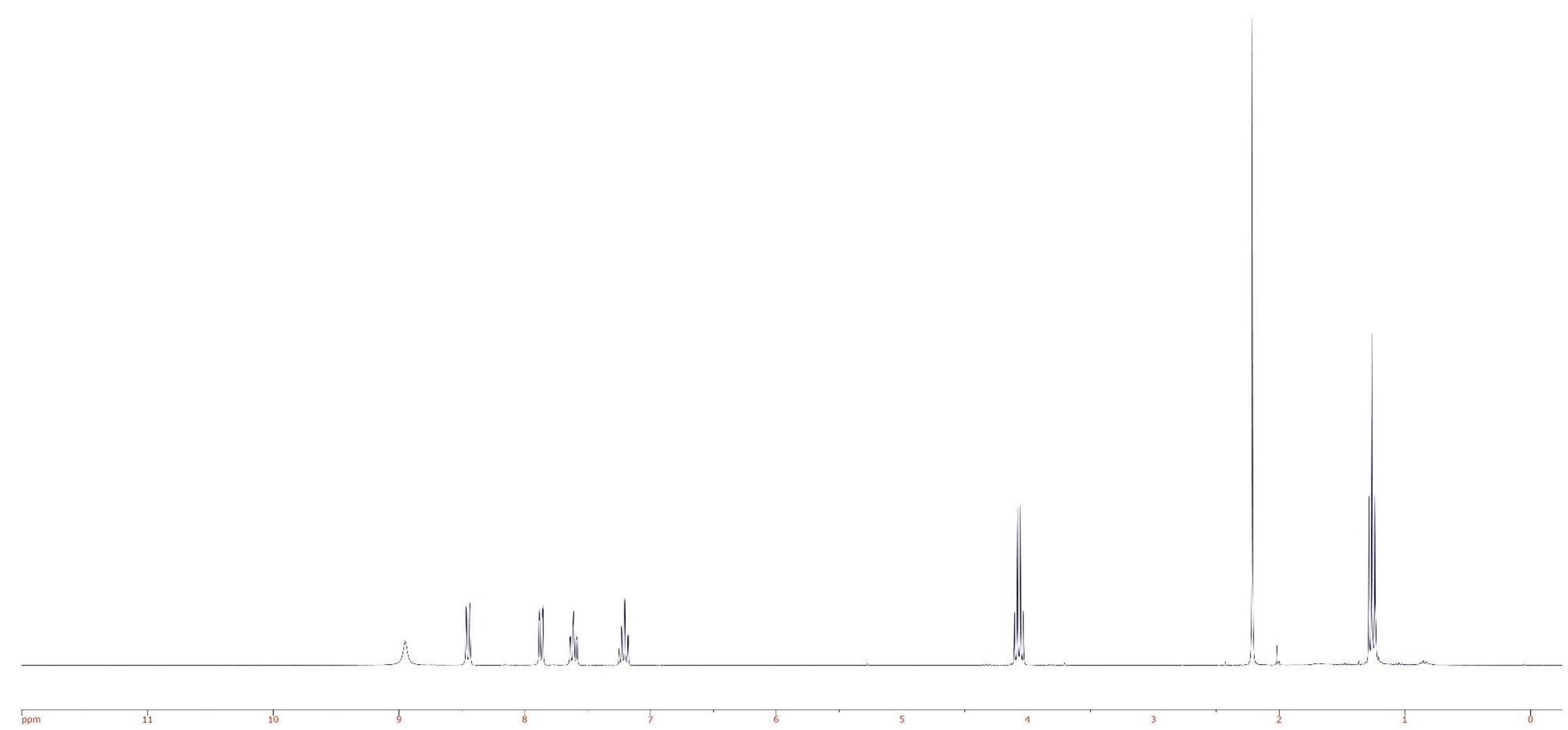




$$
\overbrace{\mathrm{NH}}^{\lambda_{\mathrm{Ne}}^{\prime 1}=0}
$$
$\gamma_{M e}$
${ }^{13}$ C NMR Spectrum

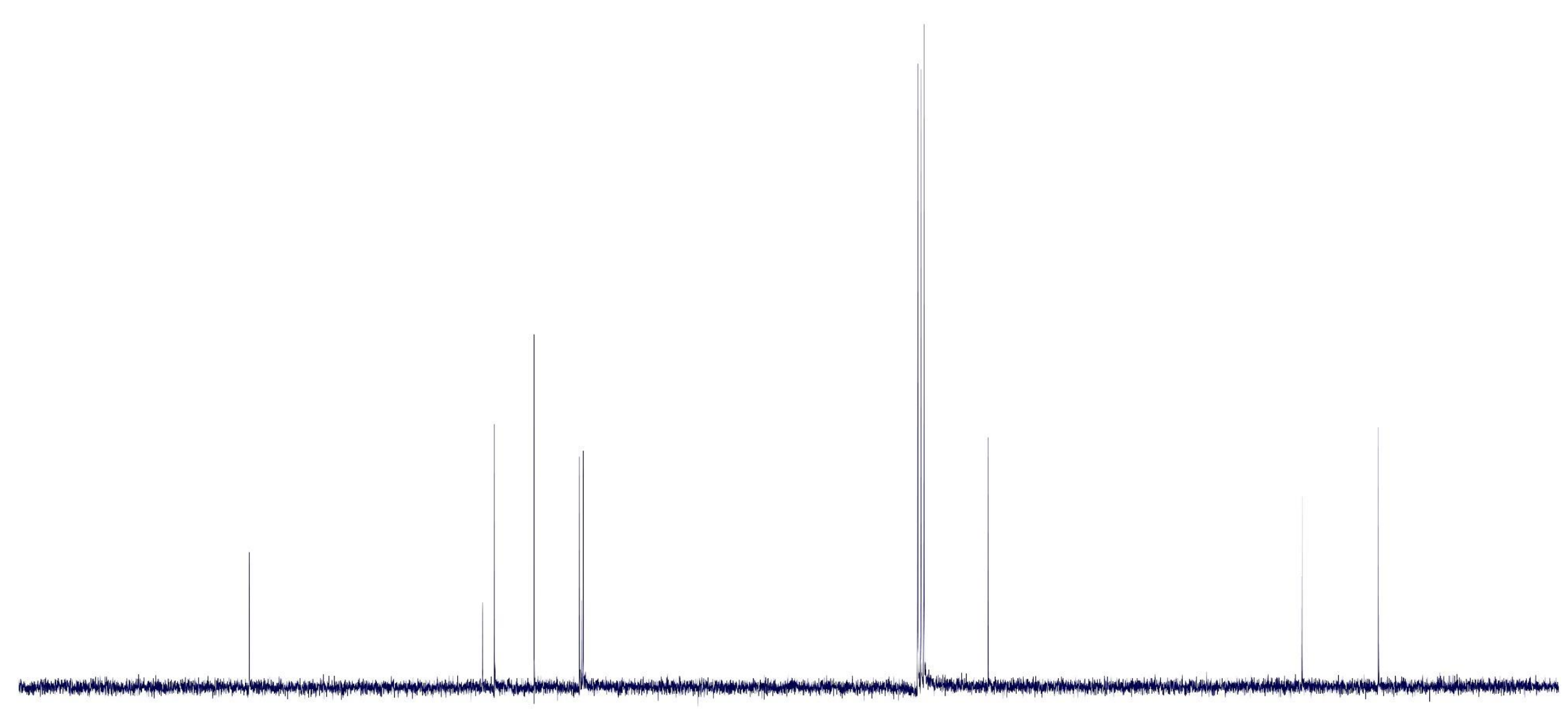




$$
\text { (H) Spectrum }
$$
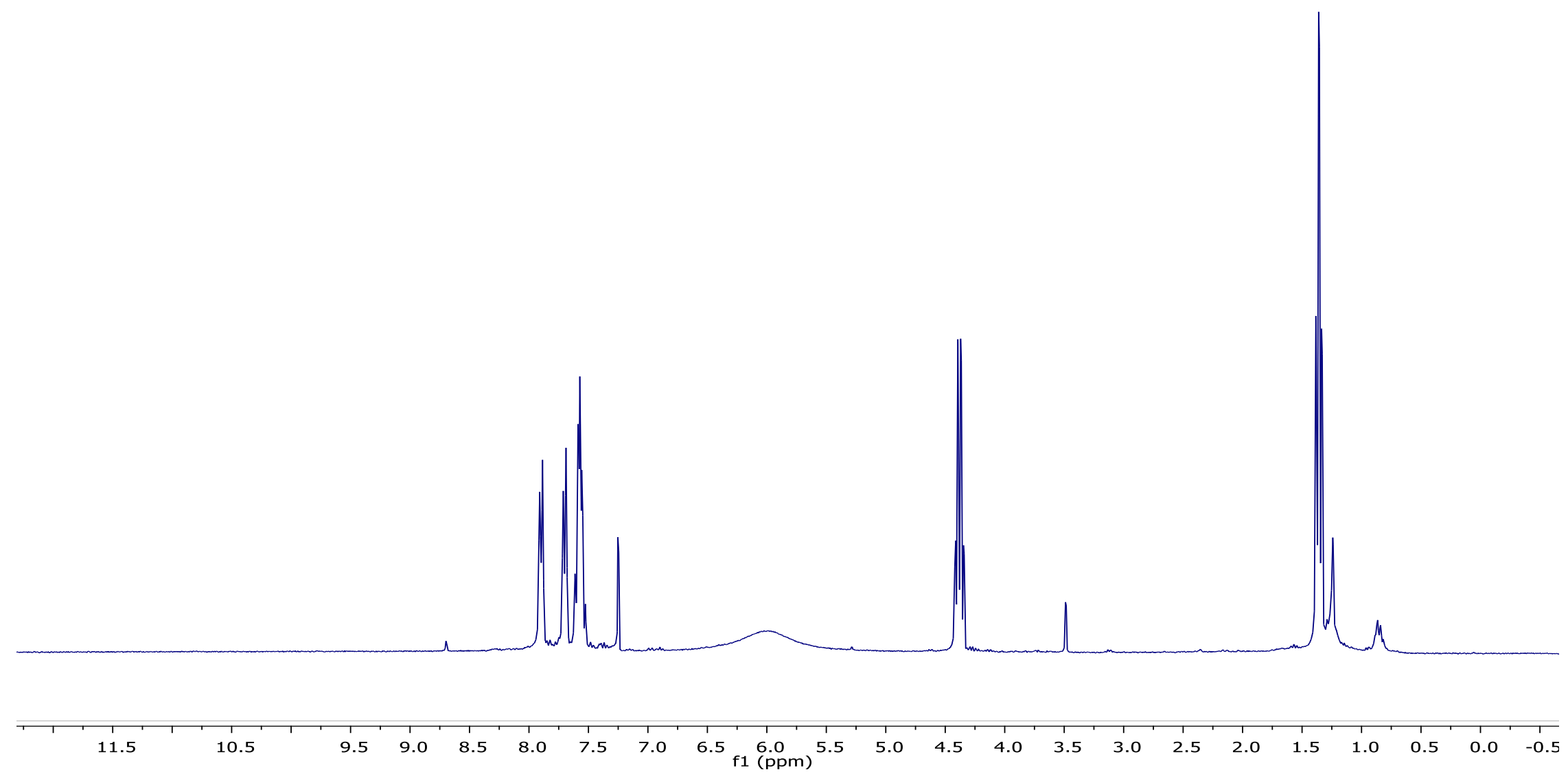


$$
\text { 贾 }
$$

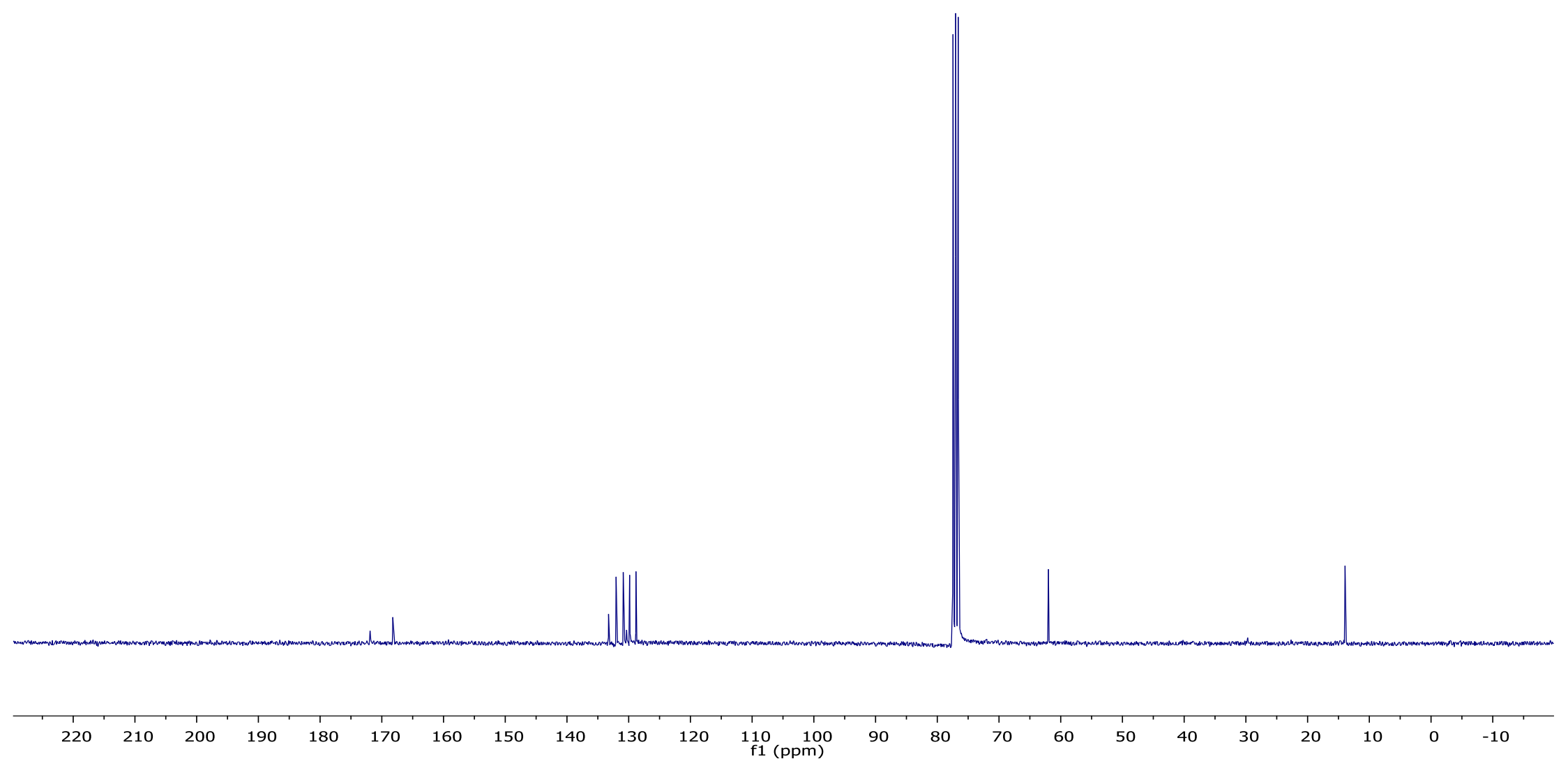


$\overbrace{O_{N}^{N H}}^{\substack{\|=0 \\ s=0}}$

H NMR Spectrum

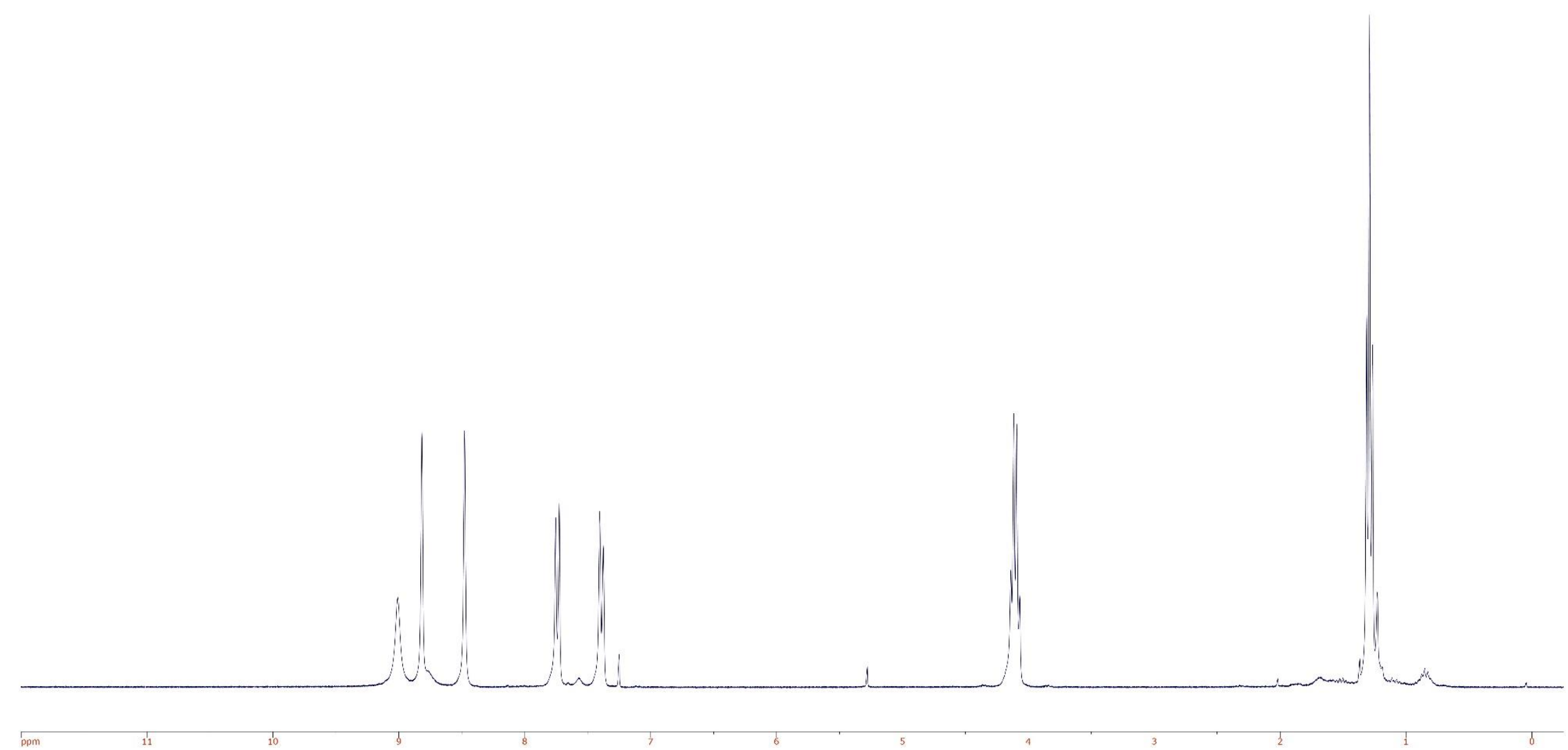


<smiles>CCOS(=O)(=O)c1ccc(Br)cc1NC=O</smiles>

${ }^{13}$ C NMR Spectrum

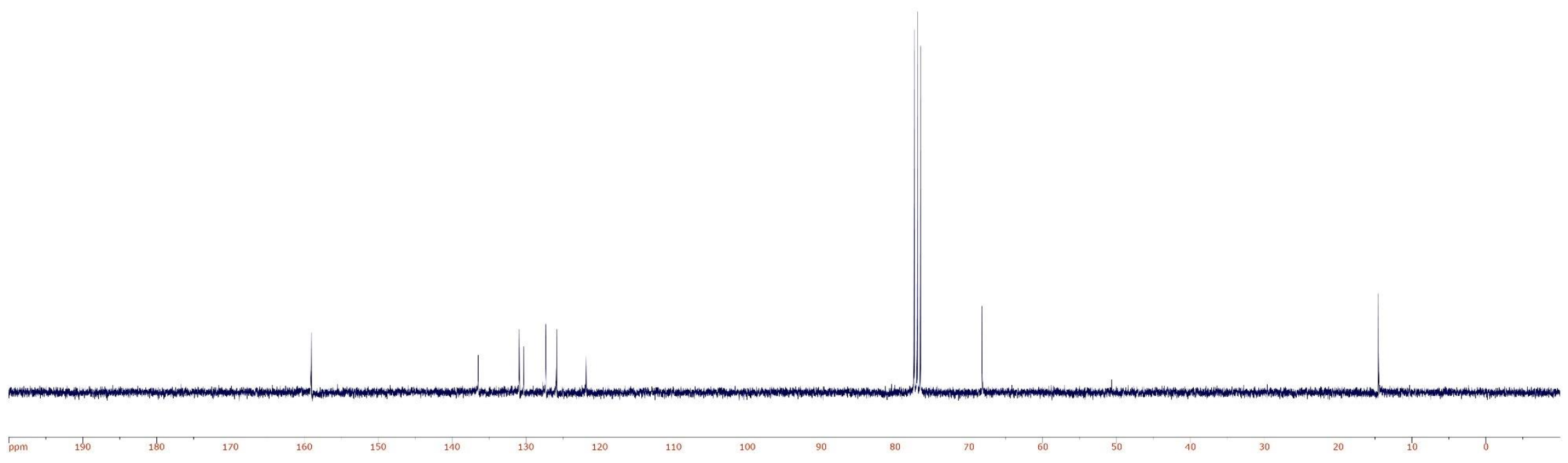

S32 


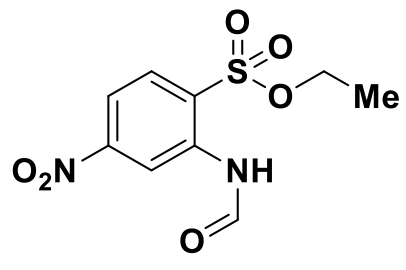

${ }^{1}$ H NMR Spectrum

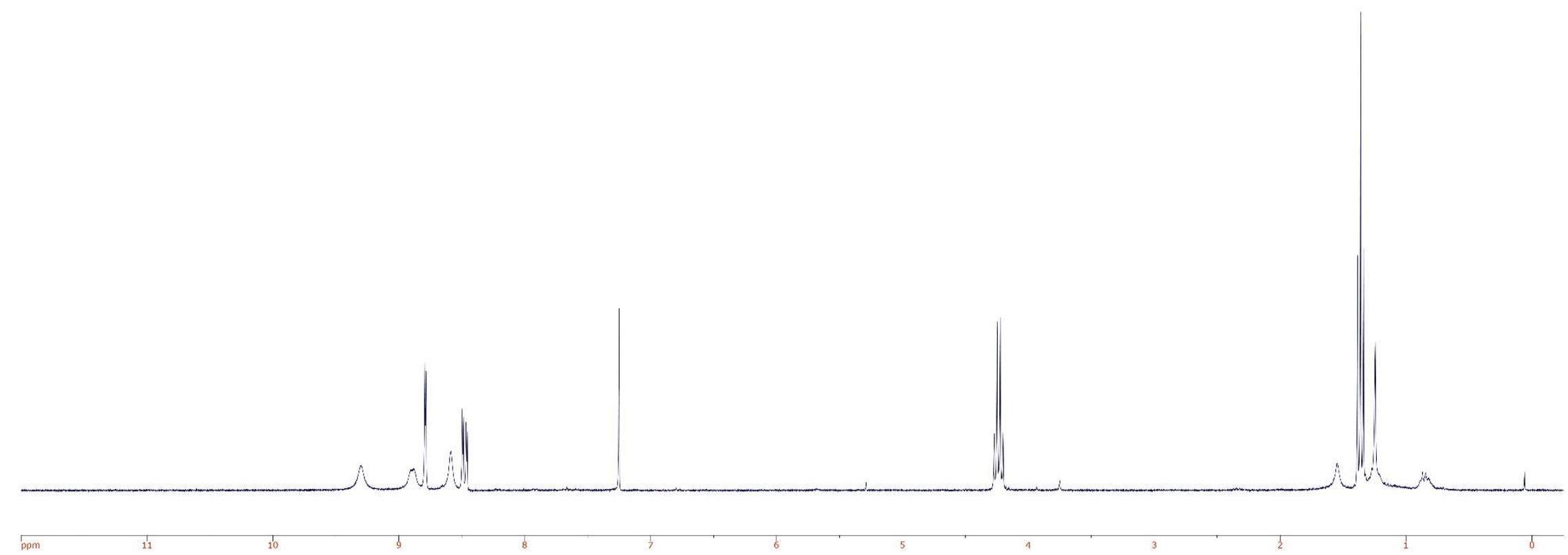




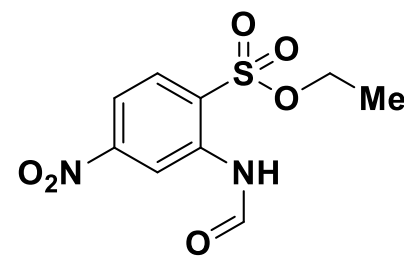

${ }^{13} \mathrm{C}$ NMR Spectrum

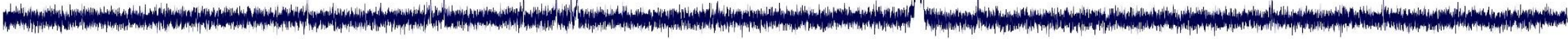
ppm $\quad 190$
$180 \cdot 170$
160
150
110
90
$70 \quad 60$
40
$30 \quad 20$ 


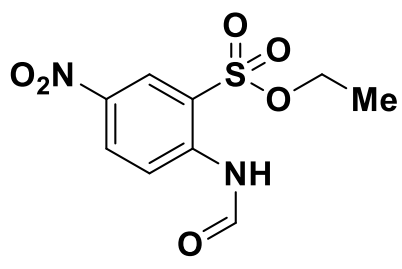

${ }^{1}$ H NMR Spectrum

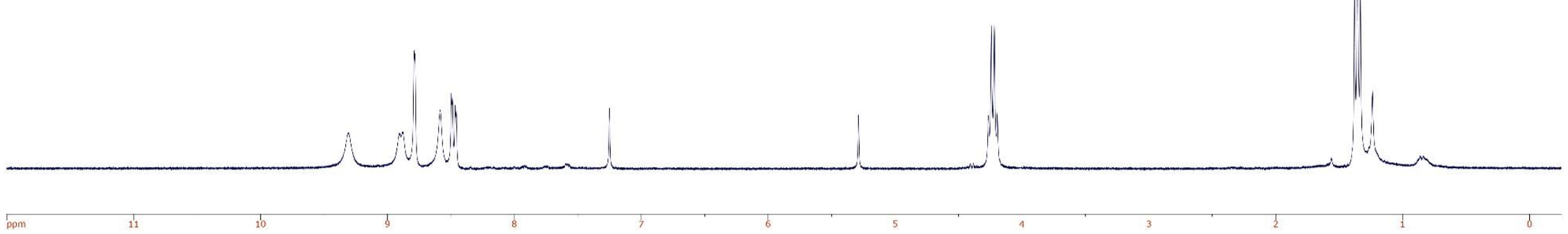




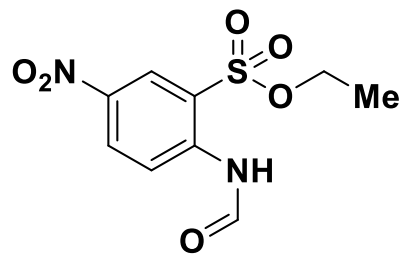

${ }^{13}$ C NMR Spectrum

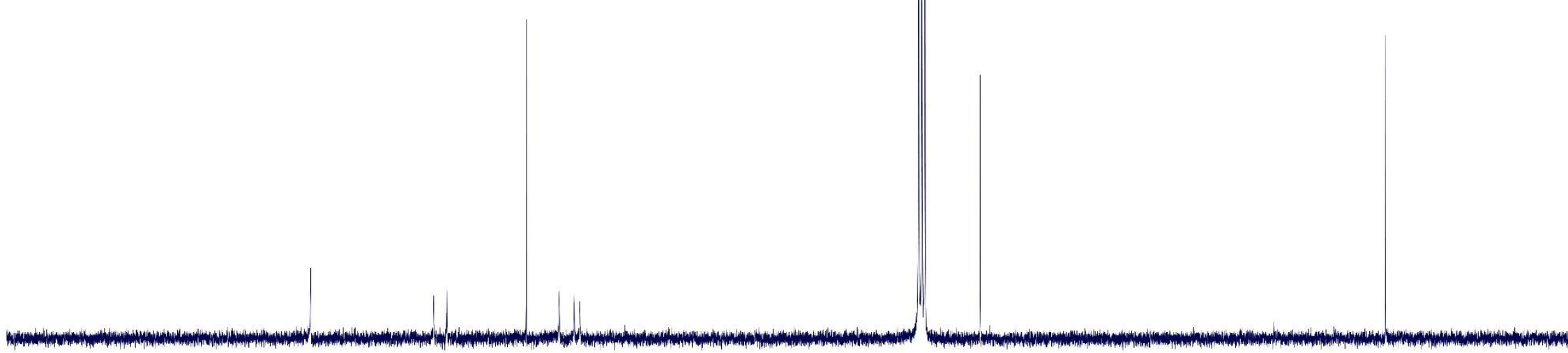




$$
\text { }
$$

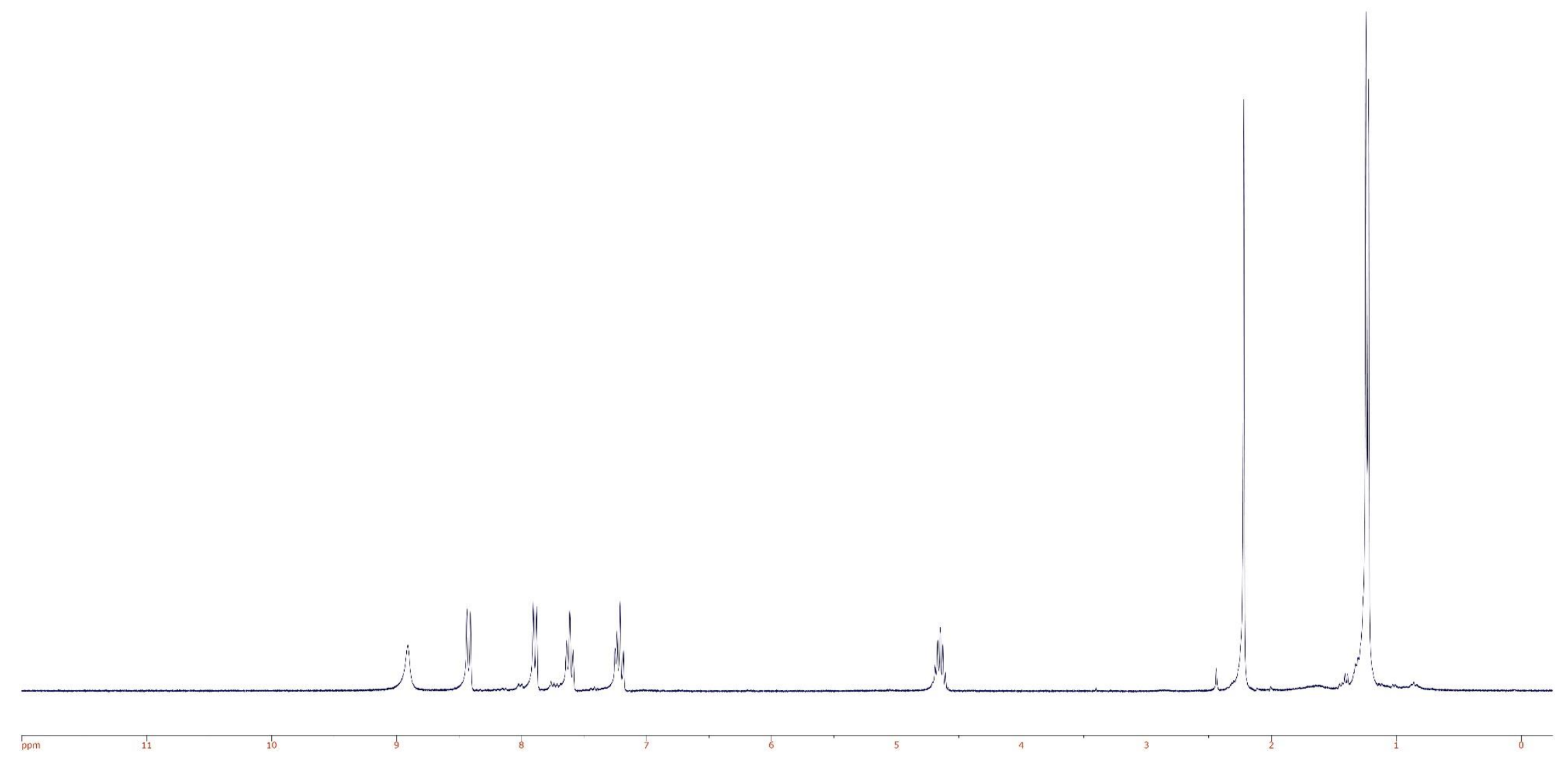




$$
{ }^{13} \mathrm{C} \text { NMR Spectrum }
$$

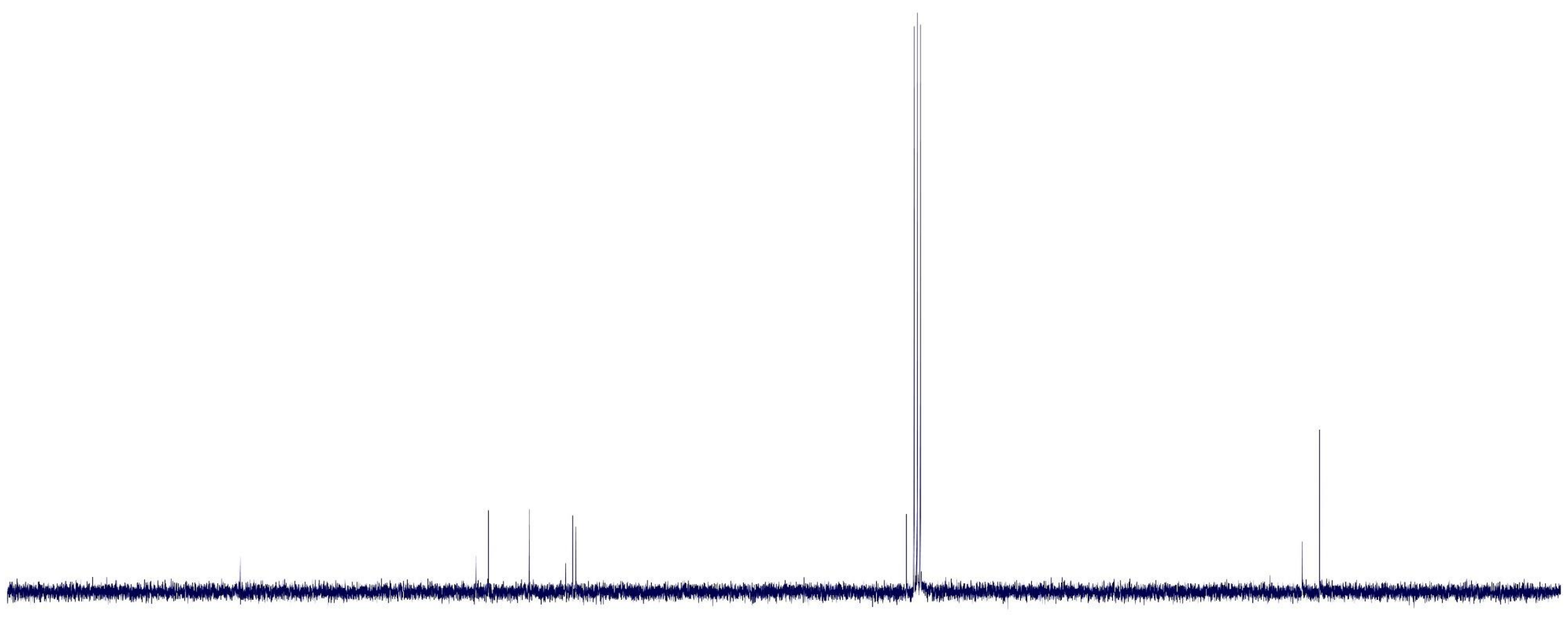




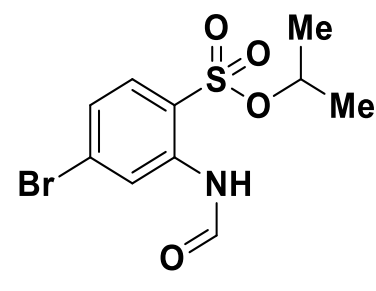

${ }^{1}$ H NMR Spectrum

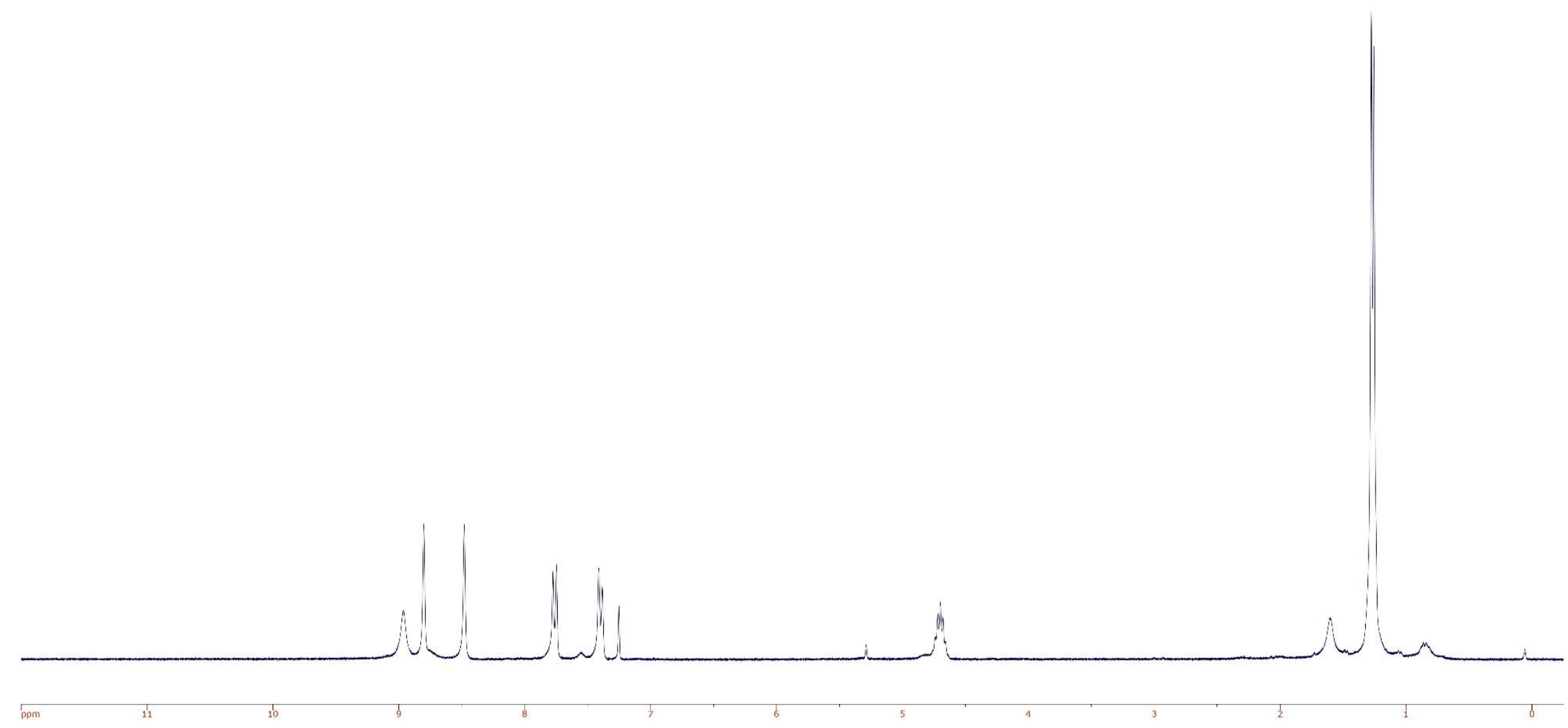



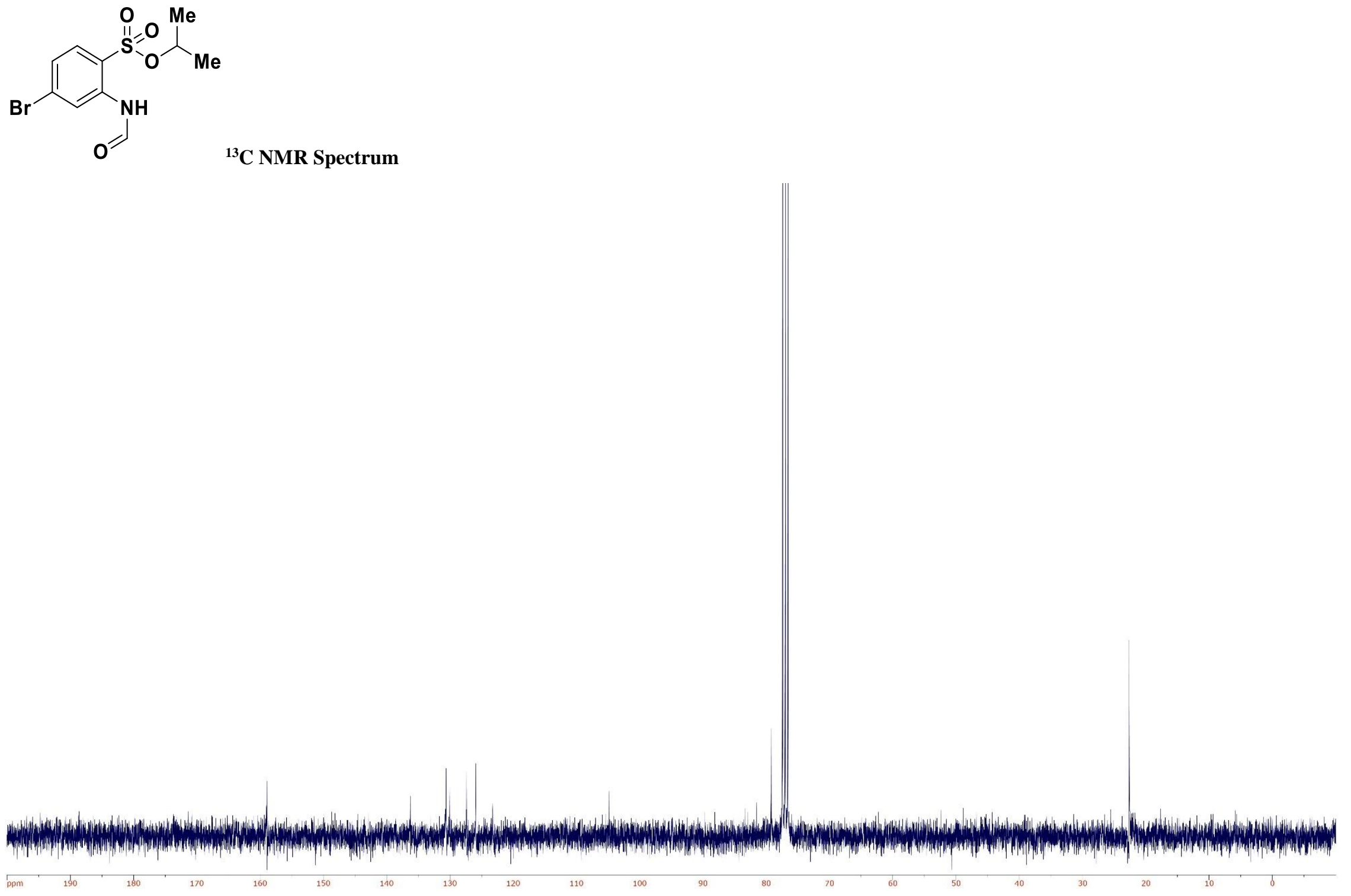